\title{
Clarin-1 gene transfer rescues auditory synaptopathy in model of Usher syndrome
}

\author{
Didier Dulon, ${ }^{1,2}$ Samantha Papal, ${ }^{1,3,4}$ Pranav Patni,, ${ }^{1,3,4}$ Matteo Cortese, ${ }^{1,3,4}$ Philippe F.Y. Vincent, ${ }^{1,2}$ Margot Tertrais, ${ }^{1,2}$ \\ Alice Emptoz, ${ }^{1,3,4}$ Abdelaziz Tlili, ${ }^{1,3,4}$ Yohan Bouleau, ${ }^{1,2}$ Vincent Michel, ${ }^{1,3,4}$ Sedigheh Delmaghani, ${ }^{1,3,4}$ Alain Aghaie, ${ }^{1,3,4}$ \\ Elise Pepermans, ${ }^{1,3,4}$ Olinda Alegria-Prevot, ${ }^{1,3,4}$ Omar Akil, ${ }^{5}$ Lawrence Lustig, ${ }^{6}$ Paul Avan, ${ }^{7}$ Saaid Safieddine, ${ }^{1,3,4,8}$ \\ Christine Petit, ${ }^{1,3,4,9}$ and Aziz El-Amraoui ${ }^{1,3,4}$
}

'UMRS 1120, Institut National de la Santé et de la Recherche Médicale (INSERM), Paris, France. université de Bordeaux, Laboratoire de Neurophysiologie de la Synapse Auditive, Bordeaux Neurocampus, Bordeaux, France. ${ }^{3}$ Unité de Génétique et Physiologie de l'Audition, Institut Pasteur, Paris, France. ${ }^{4}$ Sorbonne Universités, Complexité du Vivant, Paris, France. ${ }^{5}$ Department of Otolaryngology-Head and Neck Surgery, UCSF, San Francisco, California, USA. Department of Otolaryngology-Head and Neck Surgery, Columbia University Medical Center, New York, New York, USA. 'Laboratoire de Biophysique Sensorielle, Faculté de Médecine, Université d’Auvergne; Biophysique Médicale, Centre Jean Perrin, Clermont-Ferrand, France. ${ }^{8}$ Centre National de la Recherche Scientifique, Paris, France. ${ }^{9}$ Collège de France, Paris, France.

\begin{abstract}
Clarin-1, a tetraspan-like membrane protein defective in Usher syndrome type IIIA (USH3A), is essential for hair bundle morphogenesis in auditory hair cells. We report a new synaptic role for clarin-1 in mouse auditory hair cells elucidated by

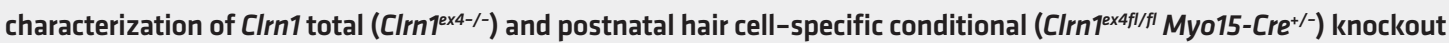
mice. Clrn 1ex4-/- mice were profoundly deaf, whereas Clrn $1^{\text {ex } 4 f / f l} \mathrm{Myo15}-\mathrm{Cre}^{+/-}$mice displayed progressive increases in hearing thresholds, with, initially, normal otoacoustic emissions and hair bundle morphology. Inner hair cell (IHC) patch-clamp recordings for the 2 mutant mice revealed defective exocytosis and a disorganization of synaptic F-actin and $\mathrm{Ca}_{v} 1.3 \mathrm{Ca}^{2+}$ channels, indicative of a synaptopathy. Postsynaptic defects were also observed, with an abnormally broad distribution of AMPA receptors associated with a loss of afferent dendrites and defective electrically evoked auditory brainstem responses. Protein-protein interaction assays revealed interactions between clarin-1 and the synaptic $\mathrm{Ca}_{\mathrm{v}}{ }^{1.3} \mathrm{Ca}^{2+}$ channel complex via the $\mathrm{Ca}_{\mathrm{v}} \boldsymbol{\beta}_{2}$ auxiliary subunit and the PDZ domain-containing protein harmonin (defective in Usher syndrome type IC). Cochlear gene therapy in vivo, through adeno-associated virus-mediated Clrn1 transfer into hair cells, prevented the synaptic defects and durably improved hearing in Clrn $1^{\text {exaflffl }} \mathrm{Myo15-C} \mathrm{Cr}^{+/-}$mice. Our results identify clarin-1 as a key organizer of IHC ribbon synapses, and suggest new treatment possibilities for USH3A patients.
\end{abstract}

\section{Introduction}

Usher syndrome type IIIA (USH3A), characterized by progressive hearing loss and retinitis pigmentosa, is caused by mutations in CLRN1 encoding clarin-1 (refs. 1, 2, and Figure 1A). CLRN1 transcripts predict several isoforms (3). At least 3 splice isoforms have been reported; reverse transcriptase PCR (RT-PCR) and Western blots on the inner ear and eye revealed the expression of isoforms 2 (containing exons 1,3 , and 4 ) and 3 (containing exons 1 and 4) (Figure 1, A and B). All the known CLRN1 mutations are located in the 3 exons of isoform 2 (1-5). CLRN1 isoform 2 encodes a 232amino acid tetraspan-like glycoprotein $(1,4,6)$. Studies of mutant mice lacking exon 1 of $\operatorname{Clrn} 1(7,8)$ and knockdown approaches in zebrafish $(9,10)$ have revealed structural and functional defects of F-actin-enriched stereocilia in hair cells. Additional roles for clarin-1 have also been sought, as mouse Clrn1 transcripts have been detected in both hair cells and the auditory primary neurons, from embryonic day 16 (E16) to adult stages $(1,11)$. Clarin-1 has

Authorship note: DD, CP, and AEA are co-senior authors. SP, PP, MC, and PFYV contributed equally to this work.

Conflict of interest: The authors have declared that no conflict of interest exists.

Submitted: March 31, 2017; Accepted: May 17, 2018.

Reference information: / Clin Invest. 2018;128(8):3382-3401.

https://doi.org/10.1172/JCI94351. also been detected at the apical and basal synaptic poles of hair cells and photoreceptor cells in the zebrafish $(9,12)$. The prolonged peak and interpeak latencies of auditory evoked brainstem responses in $\mathrm{Clrn1}^{-/-}$mice lacking exon 1 suggest a delayed activation of auditory primary neurons and transmission along the central auditory pathway (7). The amino acid sequence of clarin-1 displays some similarity to that of the $\mathrm{Ca}^{2+}$ channel subunit protein CACNG2 (stargazin) (1), a tetraspan protein involved in postsynaptic AMPA receptor trafficking and clustering in the postsynaptic active zone $(13,14)$. Together, these data suggest a possible function of clarin- 1 in hair cell synaptic transmission, a function that remains to be characterized.

We investigated the possible role of clarin-1 at hair cell ribbon synapses, by generating 2 new clarin-1-deficient mouse models (Figure 1A): one with an early ubiquitous deletion of Clrn1 exon 4 (Clrn1 $1^{e x 4-/-}$ mice) and the other with a hair cell-specific postnatal deletion of Clrn1 exon4 (Clrn $1^{e x 4 f / f l} \mathrm{Myo15}-\mathrm{Cr}^{+/-}$mice). The elimination of exon 4, which is common to all mouse clarin-1 isoforms (refs. 1-5; see also Figure 1B), should lead to Clrn1-null mice. Synaptic defects were observed in both types of clarin-1-deficient mice. Through the combination of a comparative morphofunctional analysis of these mice and biochemical studies, we showed that clarin-1 was essential for the structural organization and function of the presynaptic $\mathrm{Ca}_{\mathrm{v}} 1.3 \mathrm{Ca}^{2+}$ channels at the inner hair cell 
A Production of $C / r n 1^{-f l o x e d}\left(C / r n 1^{\text {extil/fil }}\right)$ mice

Clrn1-floxed neo allele

- exon 1 exon 2 exon 3 loxp exon 4

* Location of the reported truncating mutations in USH3A

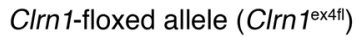

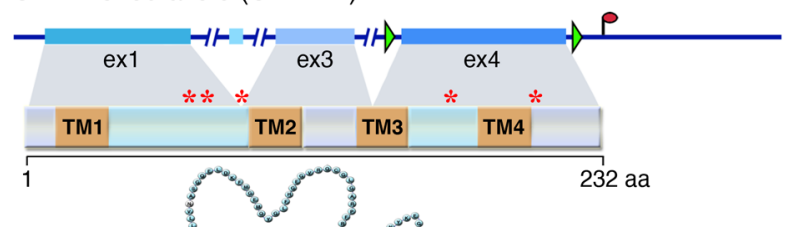

Mouse clarin-1

(isoform 2)

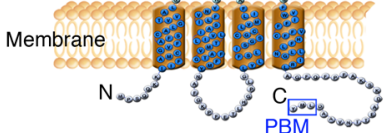

$\mathrm{PBM}$

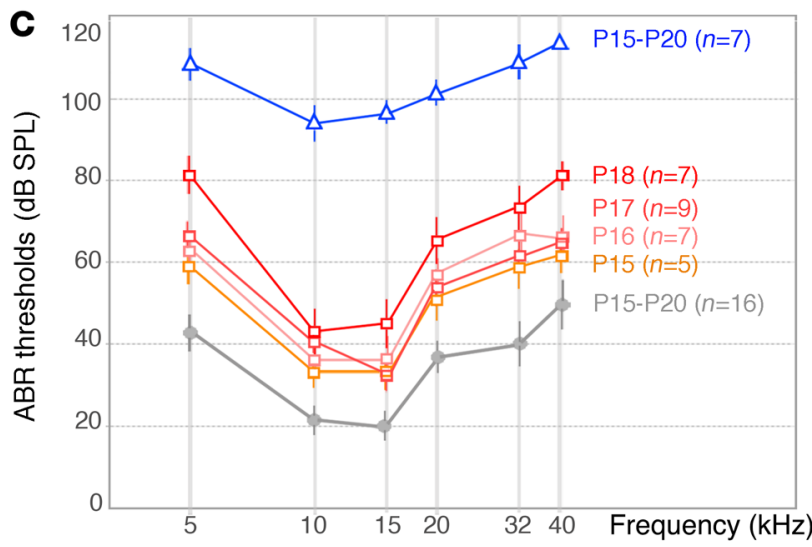

Control mice $(\bullet, \bullet, \bullet) \quad$ Clrn $1^{1 \times 4-/-}$ mice $(\Delta)$

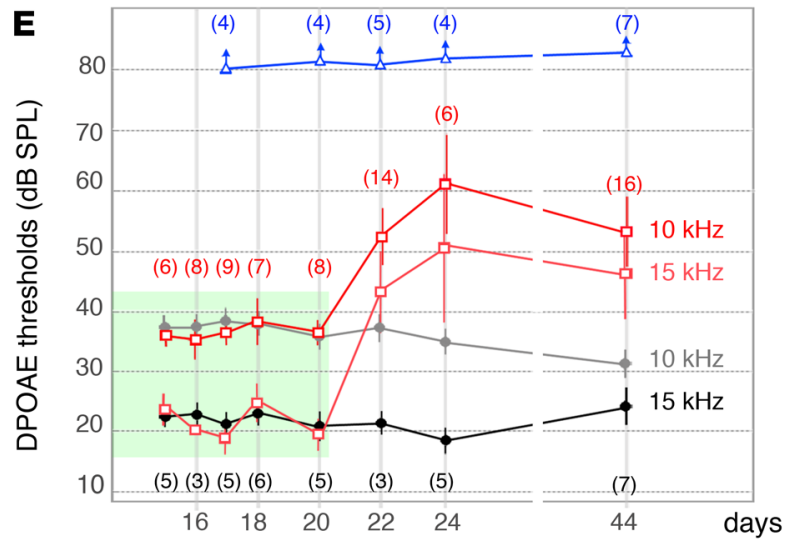

B Clarin-1 transcripts in the auditory hair cells

Clrn1 isoform 1: ex1-ex2-ex3-ex4

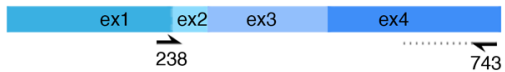

Clrn1 isoform 2: ex1-ex3-ex4
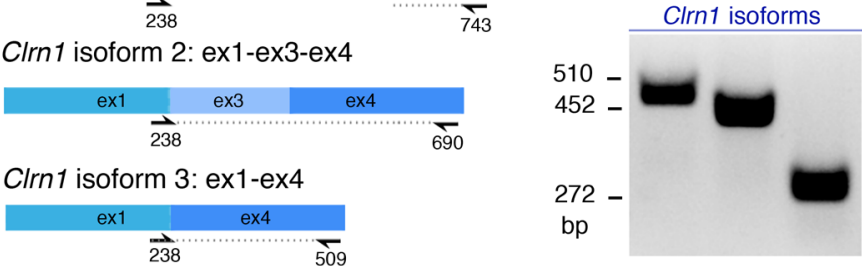

IHCs (P30 wild-type mice)

IHCs (P30 Clrn1 1exflfill Myo15-Cre ${ }^{+/-}$mice)
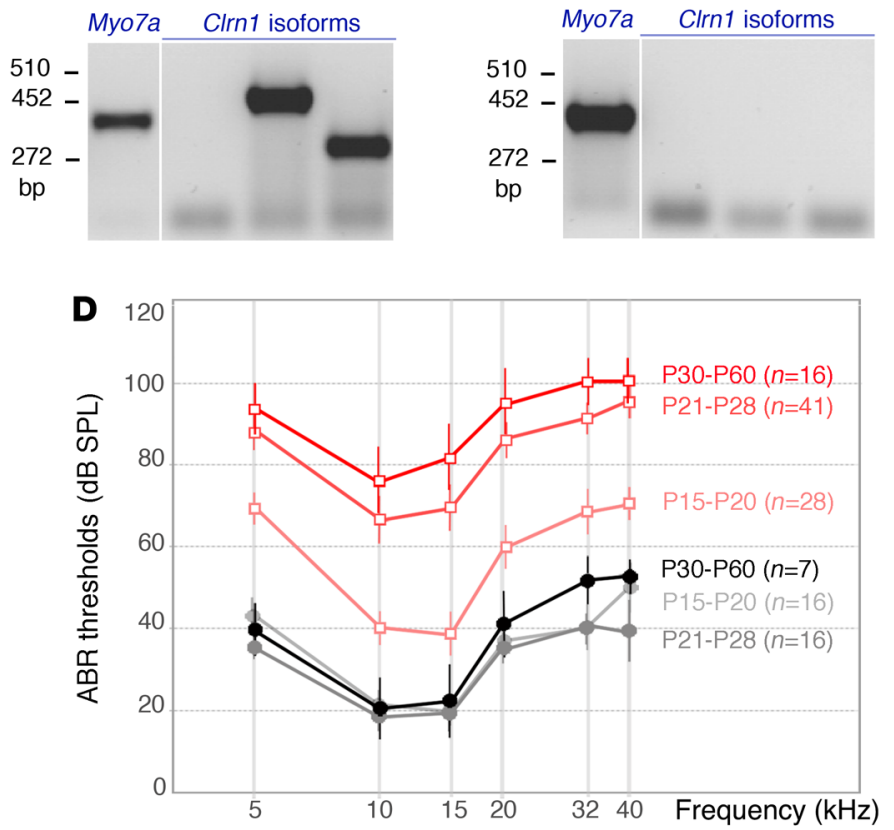

Clrntex4filfl Myo15-Cre ${ }^{+/-}$mice $(\square, \square, \square, \square)$
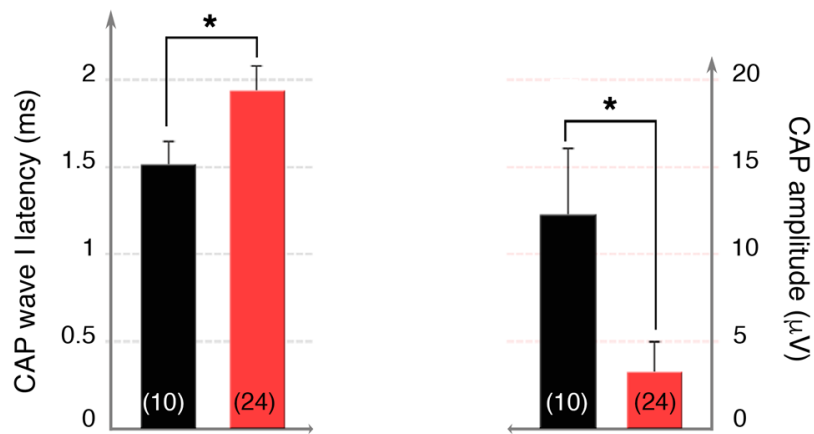

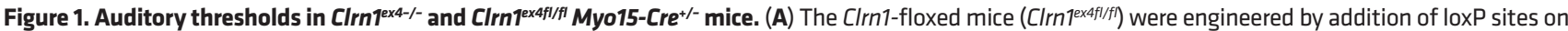

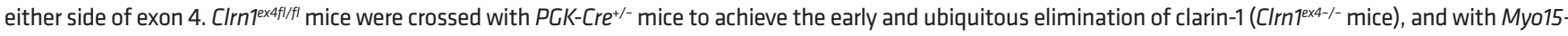
$\mathrm{Cre}^{+/-}$mice to target clarin-1 elimination specifically to hair cells at postnatal stages (Clrn pexff/fl/ Myo15-Cre ${ }^{+/-}$mice). A topological representation of the murine clarin-1, a protein containing 4 transmembrane domains with a C-terminal type II PDZ-binding motif (PBM, boxed), is shown. (B) RT-PCR shows mRNA expression of Clrn1 isoforms 1, 2, and 3 in the organ of Corti (P30) and only isoform 2 and 3 in P3O WT IHCs. Note that the expression of all isoforms is absent in P30 IHCs from

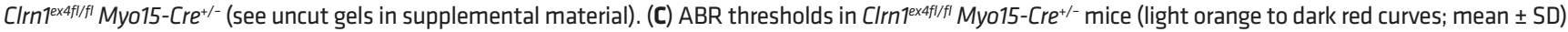

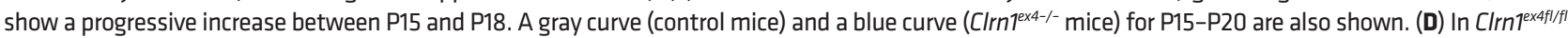
Myo15-Cre/- mice (red), ABR thresholds (mean $\pm \mathrm{SD}$ ) progressively increase to reach severe deafness at P21-P28 as compared with those in control mice (grayblack). (E) DPOAEs are absent in Clrn 7ex4-/- mice (blue) as early as P15. By contrast, Clrn 7xafl/fl Myo15-Cre ${ }^{+/-}$mice (red) display unaffected DPOAE thresholds up to P20 (green area). From P20 onward, DPOAE thresholds slowly increase by about 20-30 dB SPL. (F) Compound action potential (CAP) responses on P15-P19. CAPs were produced by $10-\mathrm{kHz}, 95-\mathrm{dB}-\mathrm{SPL}$, tone bursts. Bar charts represent CAP wave I latency and amplitude, which are significantly delayed and reduced, respectively, in Clrn ${ }^{\text {pxaff/fl }}$ Myo15-Cre ${ }^{+/-}$mice. ${ }^{*} P<0.05$ (2-tailed unpaired $t$ test with Welch's correction, $n$ in the figure). 
(IHC) ribbon synapse and, subsequently, for the distribution of postsynaptic AMPA receptors. Finally, the virus-mediated transfer of the clarin cDNA into the cochlea durably prevented synaptic defects and hearing loss in Clrn1 ${ }^{\text {ex4fl/fl }} \mathrm{Myo15}-\mathrm{Cre}^{+/-}$mice.

\section{Results}

Production ofClrn1 $1^{\text {ex4-/- }}$ and Clrn1 $1^{\text {ex } 4 f / f l}$ Myo15-Cre $e^{+/-}$knockout mice. RT-PCR showed that all 3 Clrn1 isoforms (1, 2, and 3) were present in the auditory hearing organ on postnatal day 15 (P15) and P30, whereas only isoforms 2 and 3 were found in isolated auditory hair cells (Figure 1B). We investigated clarin-1 function by generating 2 types of clarin-1-deficient mice. The first, a mouse model with a ubiquitous embryonic Clrn1 deletion, was obtained by crossing of Clrn $1^{\text {ex } 4 f / f l}$ mice, in which exon 4 , the exon common to all clarin-1 isoforms, is floxed (Figure 1A), with $\mathrm{PGK}-\mathrm{Cre}^{+/-}$mice (15). The resulting total knockout mice are referred to hereafter as Clrn $1^{e x 4-/}$ mice. The second, a mouse model with a postnatal Clrn1 deletion specific to hair cells, was generated by crossing of Clrn $1^{e x 4 f / f l}$ mice with $\mathrm{Myo15}^{-\mathrm{Cre}^{+/-}}$mice $(16,17)$. The use of $\mathrm{Myo15}_{-} \mathrm{Cre}^{+/-}$mice allowed the expression of the Cre recombinase in all cochlear hair cells, starting from birth at the base of the cochlea and at P6 at the apex (16). Using P15 ROSA26+/Myo15-Cre ${ }^{+/-}$mice and lacZ expression, we confirmed that Cre was expressed in hair cells, but not in spiral ganglion neurons (Supplemental Figure 1A; supplemental material available online with this article; https://doi.org/10.1172/JCI94351DS1). The lack of Clrn1 expression in the hair cells of Clrn1 ${ }^{\text {ex4fl/fl }} \mathrm{Myo15}-\mathrm{Cre}^{+/-}$ mice was confirmed by RT-PCR on isolated auditory hair cells on P15 and P30 (Figure 1B). These conditional clarin-1 knockout mice are referred to as Clrn $1^{e x 4 f / f l} \mathrm{Myo15}-\mathrm{Cr}^{+/-}$mice. The results of structural and functional analyses were similar for Clrn $1^{\text {ex } 4 f / f l}$, Clrn $n^{e x+/-}$, and Clrn $1^{\text {ext+/+}}$ mice. We therefore used these mice indifferently as controls.

Hearing impairment in Clrn1 $1^{\text {ex4-/- }}$ and Clrn1 $1^{\text {extff/fl }}$ Myo15$\mathrm{Cre}^{+/-}$mice. We assessed auditory function by recording auditory brainstem responses (ABRs) in Clrn1 $1^{\text {ext-/ }}$ and Clrn1 $1^{\text {ex } 4 \mathrm{fl} / \mathrm{fl}}$ Myo15-Cre ${ }^{+-}$mice, from P15, just after hearing onset (P12-P13 in mice), until P60. In Clrn $1^{e x 4-/-}$ mice, ABR thresholds following tone bursts were markedly high over the entire $5-$ to $40-\mathrm{kHz}$ frequency range, from $\mathrm{P} 15$ onward, at a sound pressure level (SPL) exceeding $90 \mathrm{~dB}$, versus only 20-40 dB in control mice (Figure 1, C and D). In addition, distortion-product otoacoustic emissions (DPOAEs), which probe the activity of outer hair cells (OHCs), the mechanical amplifiers of the sound stimulus, were undetectable in these mice with a constitutive clarin-1 knockout (Figure 1E). By contrast, Clrn $1^{e x 4 f / f l} \mathrm{Myo15}-\mathrm{Cr}^{+/-}$mice displayed progressive hearing loss from P15 to P60 (Figure 1, C and D). Interestingly, at P15-P20, the ABR thresholds of these mice were 20-40 dB higher than those of control mice, for all frequencies, whereas DPOAEs were similar to those in control mice (Figure $1 \mathrm{E}$ and Supplemental Figure 1B). Hearing thresholds continued to increase with aging, reaching an SPL of 75-100 dB by P30$\mathrm{P} 60$, across the $5-$ to $40-\mathrm{kHz}$ frequency spectrum (Figure 1, C and D). From P2O onward, DPOAE thresholds also progressively increased, to an SPL 20-30 dB above that of control mice on P44 (Figure 1E), suggesting the occurrence of an additional late pro-

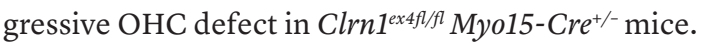

Abnormal hair bundle development in the cochlear hair cells of Clrn1 $1^{\text {ext-/- }}$ but not Clrn1 ${ }^{\text {exuff/fl }} \mathrm{Myo15-Cr} \mathrm{C}^{+/-}$mice. We investigated the cause of the hearing defect in the 2 clarin-1-deficient mice, by performing morphological analyses on the hearing organ. Scanning electron microscopy analyses in Clrn $1^{\text {ex4-/- }}$ mice, from PO onward, revealed the presence of severe abnormalities throughout the cochlea, affecting both IHC and OHC hair bundles (Figure 2, B and $\mathrm{E}$ ). In place of their normal $\mathrm{V}$ shape, the OHC hair bundles had a wavy, hooked form, and were occasionally fragmented into 2 or 3 clumps of stereocilia (Figure 2, A, B, D, and F, and Supplemental Figure 2, A and B). On P12, the short row of stereocilia had almost entirely regressed in both the IHCs and the OHCs of Clrn $1^{\text {ex4-// }}$ mice (Figure 2, B and E, and Supplemental Figure 2B). These abnormalities were observed throughout the cochlea, at its base, middle, and apex, consistent with the profound hearing loss in Clrn1ex4-/mice. By contrast, similar analyses in Clrn1 ${ }^{\text {ex } 4 f / f l} \mathrm{Myo15}-\mathrm{Cr} \mathrm{C}^{+/-}$mice showed that the IHC and $\mathrm{OHC}$ hair bundles were normal in shape until P30, with the typical 3 graded rows of stereocilia (Figure 2, A, C, D, and F, and Supplemental Figure $2 \mathrm{C}$ and Supplemental Figure 3A). On P30, in both Clrn1 $1^{\text {ex4-/- }}$ and Clrn1 ${ }^{\text {exffl/fl }} \mathrm{Myo15}^{-\mathrm{Cr}^{+/-}}$mice, the coupling between OHCs and the overlying tectorial membrane was preserved, as indicated by the presence of imprints of the tallest row of stereocilia on the lower surface of the tectorial membrane and the normal distribution of stereocilin associated with these imprints (Figure 2G and Figure 3, A and B). However, in Clrn1 $1^{e x 4 f / / f l} \mathrm{Myo15}-\mathrm{Cre}^{+/-}$mice, from P30 onward, the stereocilia of the short row were heterogeneous in length, with some missing entirely, in both the IHCs and the OHCs. The stereocilia progressively regressed until the hair bundles degenerated completely, as observed on P120 (Supplemental Figure 3, A and B).

We explored the distribution of the USH proteins of the mechano-electrical transduction machinery (18) in the hair bundles of clarin-1-deficient mice (Figure 3, A and B). The apical region of the stereocilia was typically enriched in USH1 proteins (myosin VIIa, harmonin, cadherin-23, and protocadherin-15) in Clrn1 $1^{\text {ext-/- }}$ (Figure 3, A and B) and Clrn1 ${ }^{\text {ex4ff/fl }}$ Myo15-Cre ${ }^{+/-}$(Supplemental

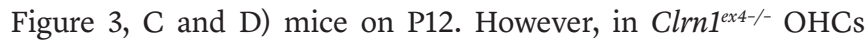
with fragmented hair bundles, the staining of the F-actin-labeled cuticular plate on the apical surface was heterogeneous, with furrows corresponding to regions of clumped stereocilia (Figure 3C). By contrast, F-actin staining was homogeneous in Clrn1 $1^{\text {ex } 4 \mathrm{fl} / \mathrm{fl}}$ Myo15-Cre ${ }^{+/-}$mice, in which all the OHCs had normal V-shaped hair bundles and a normal cuticular plate, regardless of the stage or cochlear position analyzed (Figure 3D).

An early defect of sound-evoked auditory nerve compound action

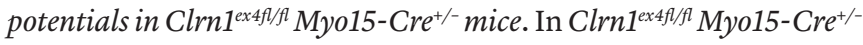
mice, the progressive increase in $\mathrm{ABR}$ thresholds in a context of normal DPOAEs and hair cell stereocilia morphology until P2O suggested that the postnatal loss of clarin-1 probably affected the normal functioning of IHCs and/or their associated primary auditory neurons. We tested this hypothesis, by recording cochlear nerve compound action potentials (CAPs), which reflect the synchronous firing of primary auditory neurons in response to neurotransmitter release at IHC ribbon synapses, in young Clrn1 $1^{\text {ex } 4 f / f l}$ Myo15-Cre ${ }^{+/-}$mice between the ages of P15 and P2O. In response to loud sound stimuli (SPL of $95 \mathrm{~dB}$ ), for which the cochlear response is independent of the amplifying activity of OHCs, CAP latencies 
A
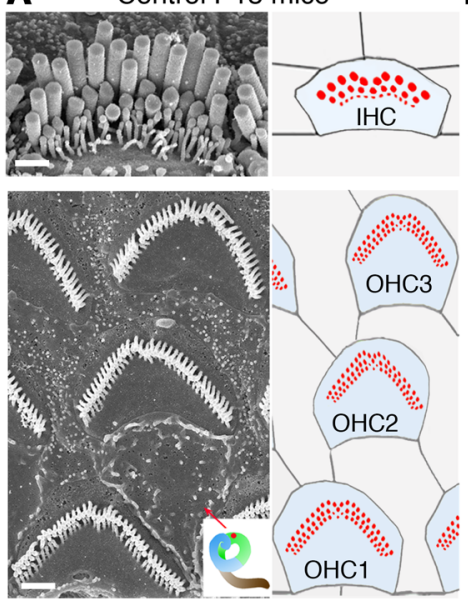

D V-shaped $\mathrm{OHC}$ hair bundle of a control mouse

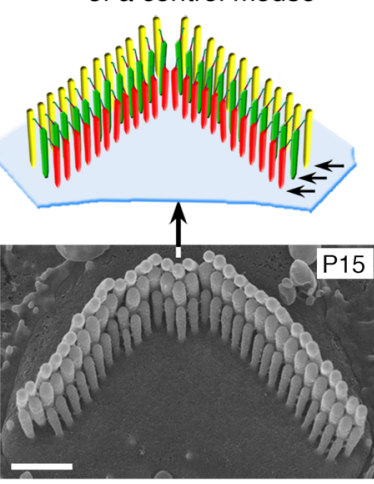

B
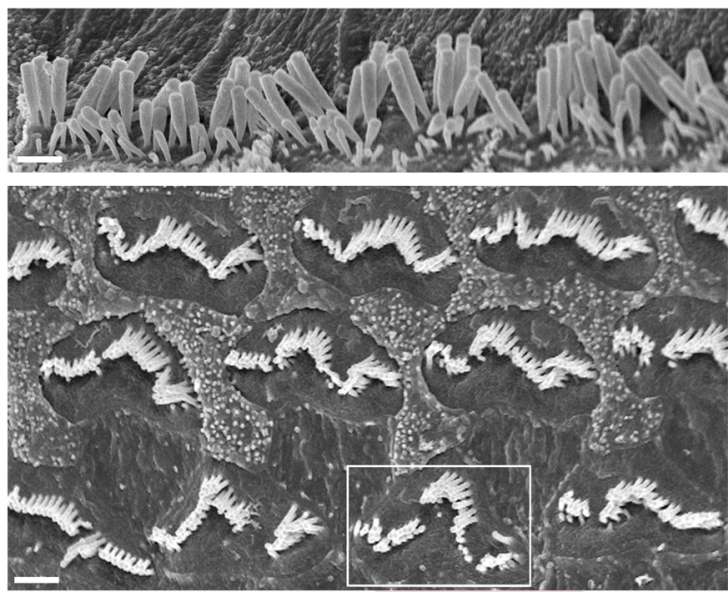

E Altered shape of $C / r n 1^{\text {ex4-- }} \mathrm{OHC}$ hair bundles
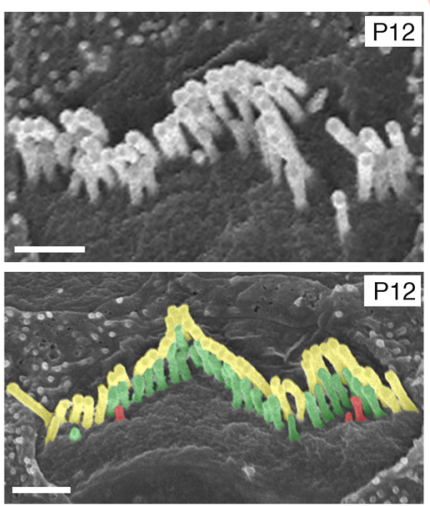
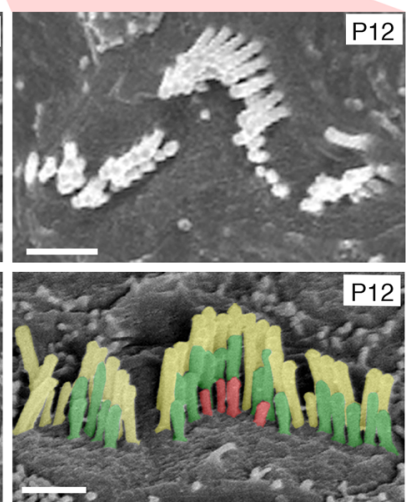

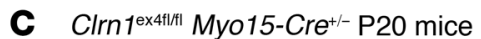
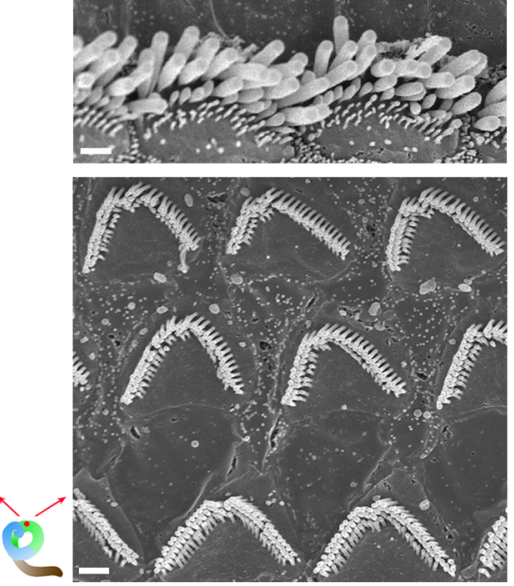

F Normal $\mathrm{OHC}$ hair bundles

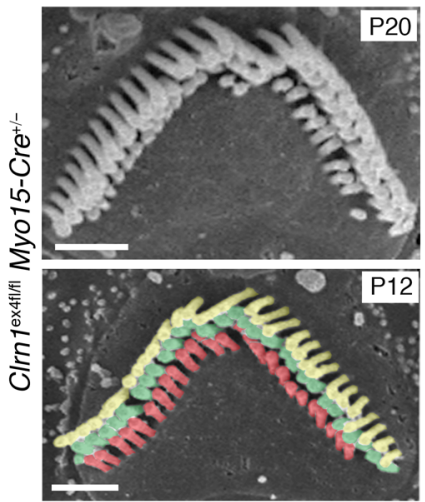

G

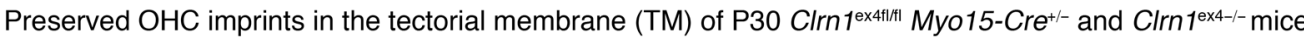
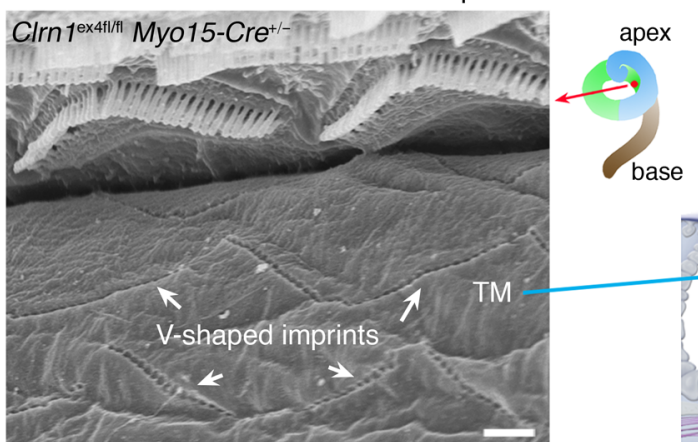

position of $\mathrm{OHC}$ imprints
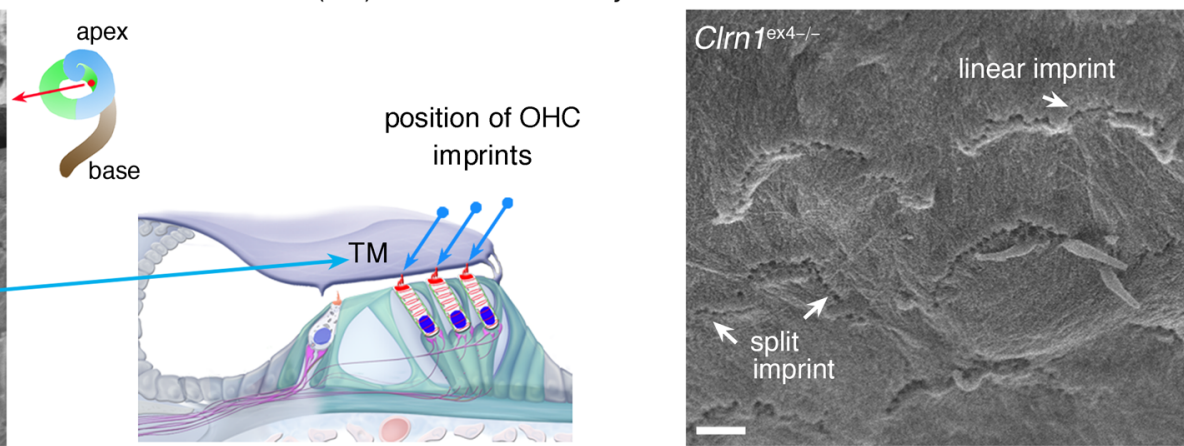

Figure 2. Architecture of the hearing organ in Clrn 1ex4-/- mice and Clrn 1exafl/fl Myo15-Cre ${ }^{+/-}$mice. (A-F) Hair bundle structure is affected in Clrn $7^{\text {ex4-/- }}$ mice

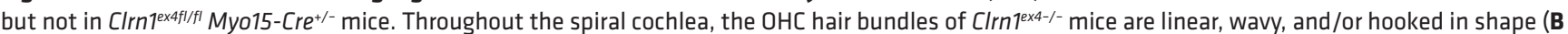

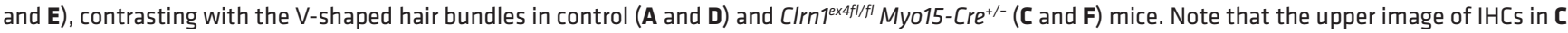
is a crop of Supplemental Figure 3A (lower portion of P2O panel). Similar hair bundle abnormalities were observed along the entire cochlear axis between P12 and P25 (images representative of 12 mice). The stereocilia of the short row (colored in red) regressed entirely in most of the OHCs of P12 Clrn1ex4-/- mice (E), but not in those of P20 Clrn 7exffl/fl Myo15-Cre ${ }^{+/-}$mice (F). (C) The physical coupling between the tallest stereocilia of OHCs and the overlying tectorial

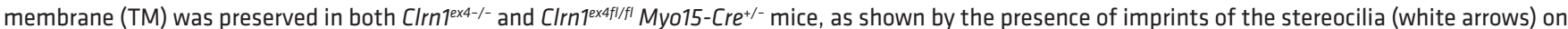
the lower surface of the tectorial membrane. Scale bars: $1 \mu \mathrm{m}$.

were $30 \%$ longer, with amplitudes $75 \%$ smaller, as compared with control mice (Figure $1 \mathrm{~F}$ ). These findings suggested a defect of synaptic transmission from IHCs to their primary afferent neurons in the absence of clarin-1.

Abnormal $\mathrm{Ca}^{2+}$ currents and lower efficiency of $\mathrm{Ca}^{2+}$-triggered synaptic exocytosis in IHCs lacking clarin-1. Synaptic vesicle exocytosis in IHCs is known to be controlled by $\mathrm{Ca}^{2+}$ influx through L-type
$\mathrm{Ca}_{\mathrm{v}} 1.3$ channels, which are tightly clustered in the vicinity of the synaptic ribbons (19-21). We investigated possible changes in the voltage-dependent activation of $\mathrm{Ca}^{2+}$ currents in mutant IHCs. As in control mice (22), we observed a transient developmental upregulation of $\mathrm{Ca}^{2+}$ current density, defining a peak $\mathrm{Ca}^{2+}$ current normalized against cell membrane area, between P6 and P9 in Clrn1 $1^{\text {ex4-/- }}$ and Clrn1 ${ }^{\text {ex4ff/fl }}$ Myo15-Cre ${ }^{+/-}$IHCs (Figure 4, A-E). On P9, 
A
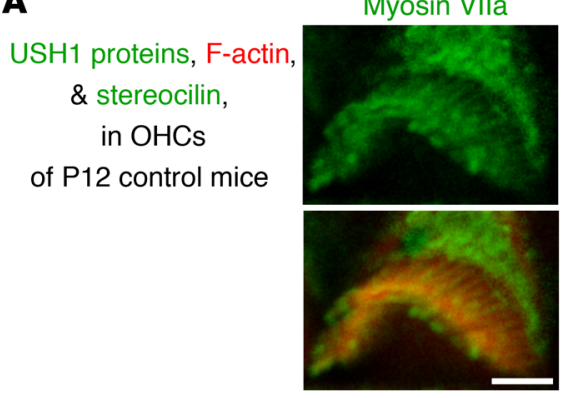

B Myosin VIla

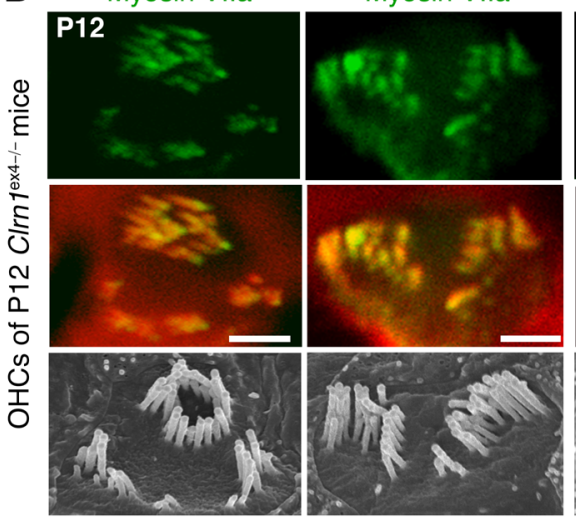

C

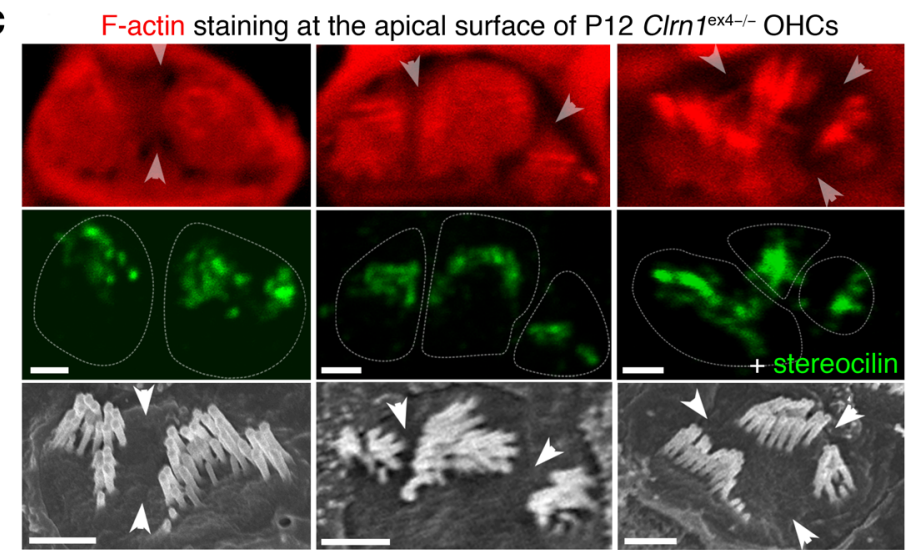

Harmonin b
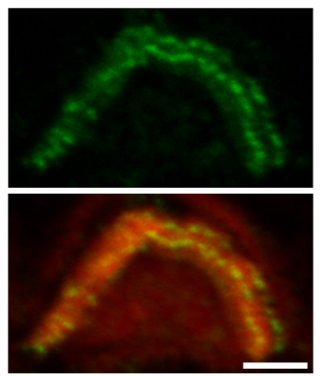

Harmonin b
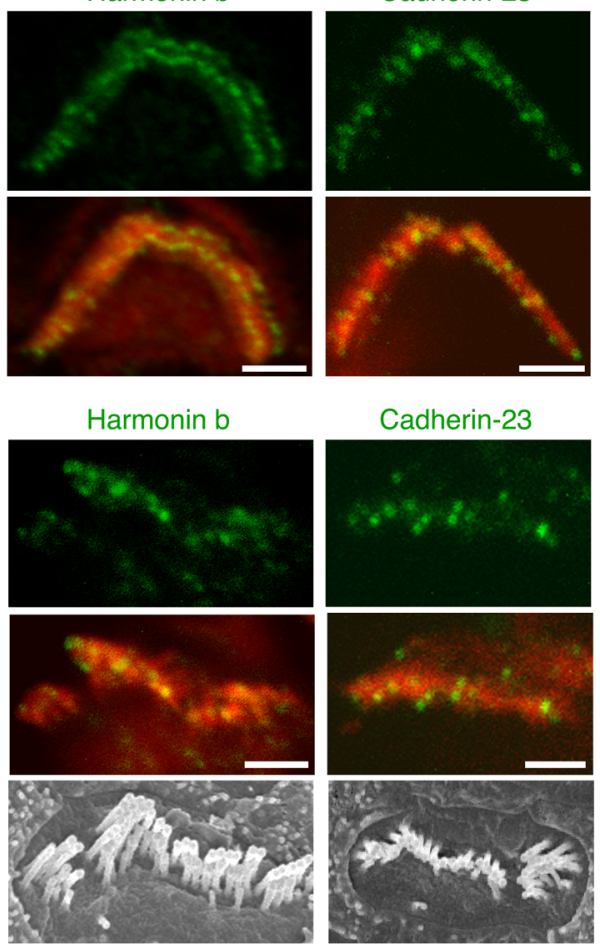

Cadherin-23
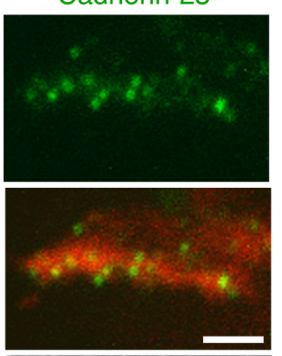

Protocadherin-15
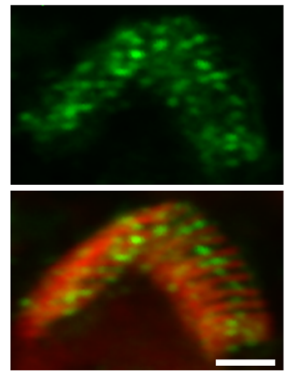

Protocadherin-15
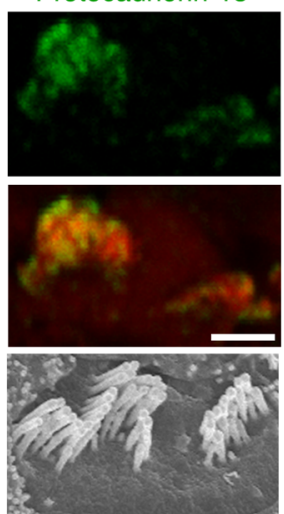

D F-actin staining at the apical surface of control and Clrn 10x4l//1/1 Myo15-Cre ${ }^{+/-} \mathrm{OHCs}$
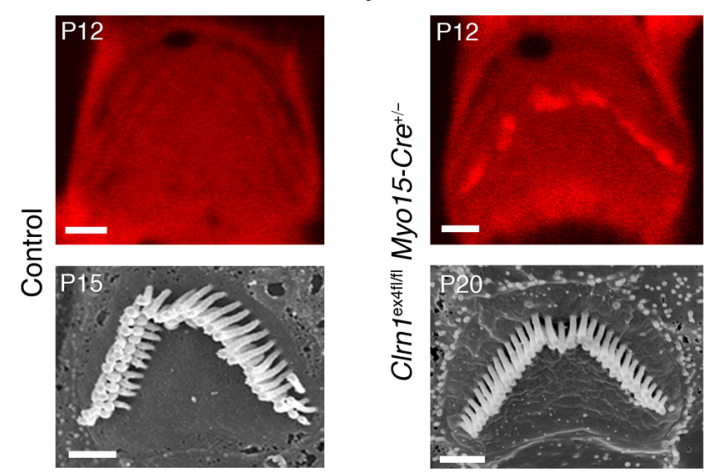

Stereocilin

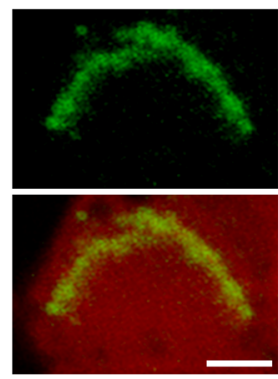

Stereocilin

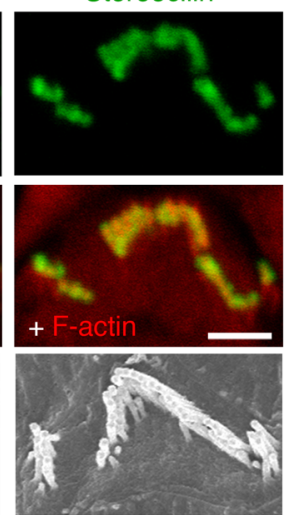

Figure 3. Distribution of F-actin and USH1 proteins in OHCs lacking clarin-1. (A) Distribution of USH1 proteins (myosin VIla, harmonin b, cadherin-23, protocadherin-15) and stereocilin in the apical region of F-actin-labeled stereocilia (red) in P12 control mice. (B) The typical targeting and localization of

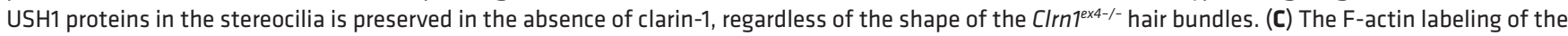
cuticular plate in P12 Clrn $7^{\text {ex4-/- }}$ mice is irregular, with the presence of furrows delimiting regions of clumped stereocilia. Stereocilin immunostaining (green)

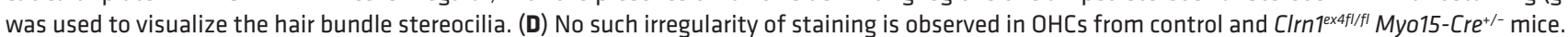

Scanning electron microscopy micrographs show selected OHC hair bundles (from P12 Clrn 1ex4-/-, P15 control, and P20 Clrn 1exfflffl Myo15-Cre ${ }^{+/-}$mice) with hair bundle shapes similar to those of the immunostained hair bundles. Images obtained from 2-5 mice for each genotype. Scale bars: $1 \mu \mathrm{m}$.

an immature stage before the onset of hearing, IHCs from both Clrn1 $1^{\text {ex4-/- }}$ and Clrn1 $1^{\text {exffl/fl }}$ Myo15-Cre ${ }^{+/-}$mice had normal $\mathrm{Ca}^{2+}$ currents, with amplitudes and densities similar to those of IHCs from control littermates (Figure 4, A, C, and E, and Supplemental Table 1). From $\mathrm{P} 13$ to $\mathrm{P} 18$, after the onset of hearing, $\mathrm{Ca}^{2+}$ current density gradually declined to steady-state levels below the values obtained on P6 in control mice, whereas a much less pronounced downregulation was observed in the IHCs of Clrn $1^{\text {ex4-/ }}$ and Clrn1 $1^{\text {ex } 4 f / f l}$ $\mathrm{Myo15}_{-} \mathrm{Cre}^{+/-}$mice (Figure 4, B, D, and E, and Supplemental Table

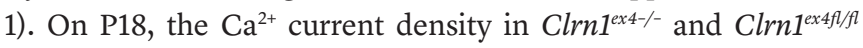
Myo15-Cre ${ }^{+/-}$mice was almost twice that in control mice. These results suggested that clarin-1 is essential for the synaptic maturation of $\mathrm{Ca}^{2+}$ channels in IHCs at the onset of hearing.

An analysis of the voltage-dependent activation of $\mathrm{Ca}^{2+}$ currents revealed a negative shift of $7 \mathrm{mV}$ in P18-P21 Clrn1 $1^{\text {ex } 4 f / f l}$ $\mathrm{Myo15}_{\mathrm{Cre}}{ }^{+-}$mice (Figure 4, F and G). This negative shift began on P9 before the occurrence of significant changes in $\mathrm{Ca}^{2+}$ current amplitude and density, and increased over subsequent days. A similar negative shift was also observed in Clrn1 $1^{\text {ext-r- }}$ mice on P9 and P13 (Supplemental Table 1). On P18, the steepness of the voltage-dependent activation curve was also significantly lower in the mutant mice $(4.2 \pm 0.1 \mathrm{pA} / \mathrm{mV}, n=7$ cells $)$ than in control mice 
A

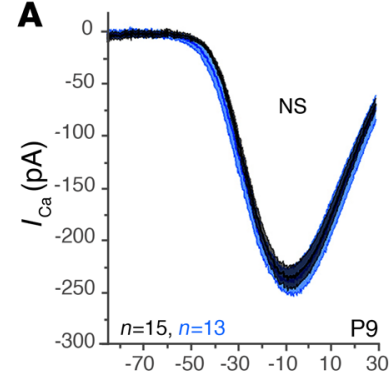

Membrane potential $(\mathrm{mV})$

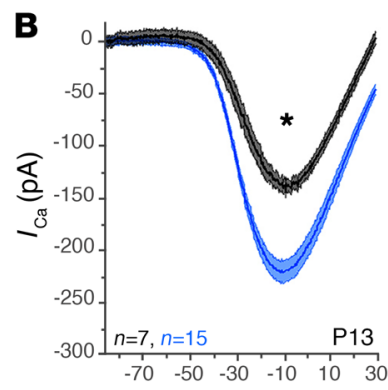

Membrane potential (mV)

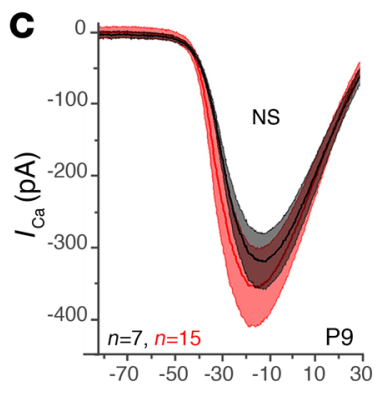

Membrane potential $(\mathrm{mV})$

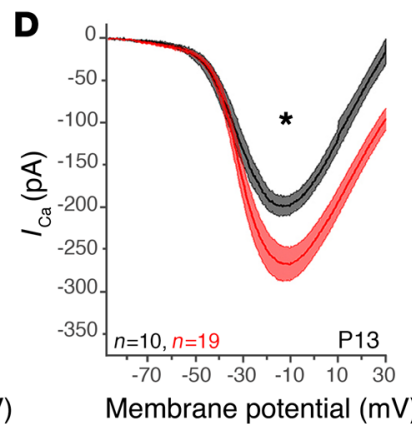

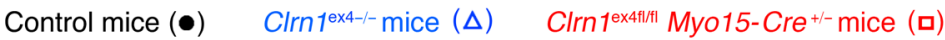
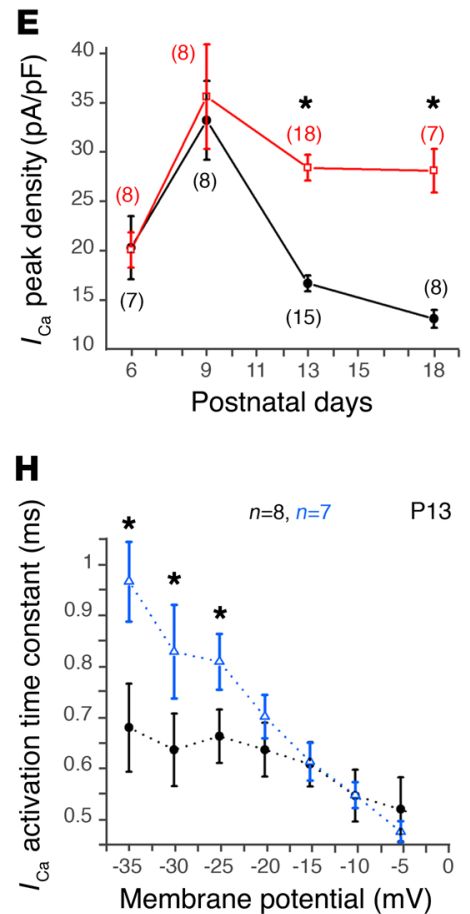

$\mathbf{F}$

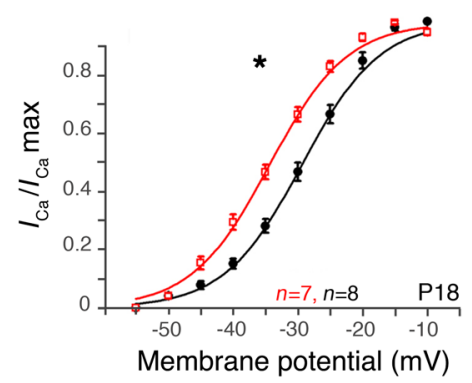

I

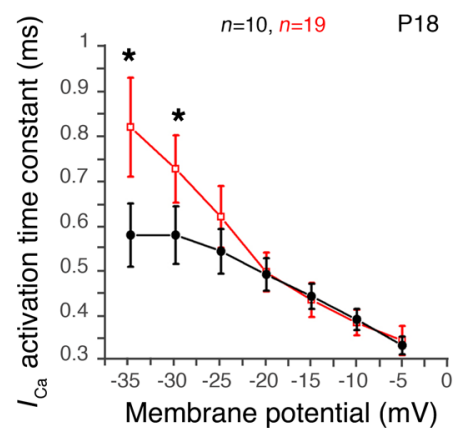

G

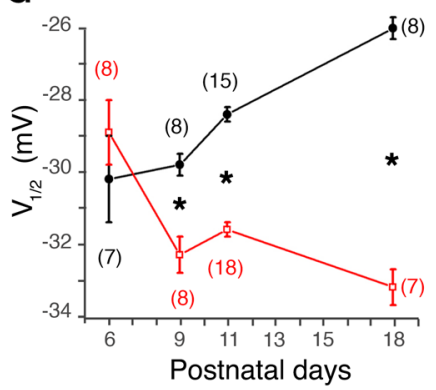

J

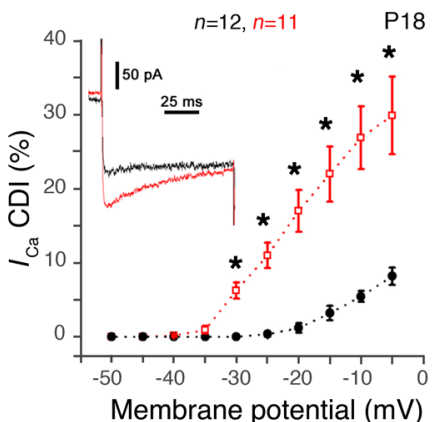

Figure 4. Abnormal $\mathrm{Ca}^{2+}$ currents in mutant IHCs lacking clarin-1. (A-D) Ca ${ }^{2+}$ currents in IHCs were activated with a depolarizing voltage-ramp protocol (1 $\left.\mathrm{mV} / \mathrm{ms}\right)$ from -90 to +30 mV. The amplitude of Ca ${ }^{2+}$ currents is normal on P9, but larger in Clrn7ex4-/- (blue curves, $\mathbf{A}$ and $\mathbf{B}$ ) and Clrmpexfi/fl Myo15-Cre ${ }^{+/-}$(red curves, C and D) mice than in controls (black curves, A-D) on P13. (E) Comparative change in peak $\mathrm{Ca}^{2+}$ current density (peak $I_{\mathrm{Ca2}_{+}}$at -10 mV normalized with respect to cell size) with age, at pre- and post-hearing stages (from P6 to P18), in IHCs from Clrn 7exffl/fl Myo15-Cre ${ }^{+/-}$mice and control mice. (F) Boltzmann fit of the I-V curve for IHC Ca ${ }^{2+}$ currents (100-ms voltage steps) in Clrn 1exff//fI $M y 015-\mathrm{Cre}^{+/-}$and control mice on P18. (C) Comparative change in the half-maximal activation voltage of $I_{\text {Ca2+ }}\left(\mathrm{V}_{1 / 2}\right)$ before and after hearing onset (from P6 to P18) in IHCs from Clrn pexaf//fI Myo15-Cre ${ }^{+/-}$and control mice. (H and I) I $I_{\text {c23 }}$ activation time constant measured for various voltage

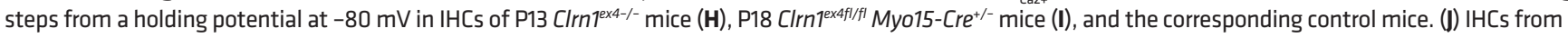
P18 Clrn 7exffl/fl Myo15-Cret/- (red) and control (black) mice were subjected to voltage steps from -80 mV to various membrane potentials for 100 milliseconds (inset: current traces for a Clrn ${ }^{\text {exffl/fl }}$ Myo15-Cre $\mathrm{Cr}^{+/-}$mouse and a control mouse). The current reduction at the end of the 100-millisecond step (Ca ${ }^{2+-d e p e n d e n t ~ i n a c t i v a-~}$ tion, CDI) is expressed as a percentage of $I_{\mathrm{Ca}+}$ peak (\%): CDI $=\left[I_{\mathrm{Ca}+\text { peak }}-I_{\mathrm{Ca}+100 \mathrm{~ms}}\right] / I_{\mathrm{Ca} 2+\text { peak }}$. The values shown are means $\pm \mathrm{SEM} .{ }^{*} P<0.05(\mathrm{E}-\mathrm{J}$, Student's $t$ test with Welch's correction; and A-D, 2-way ANOVA); $n$, number of cells.

$(5.7 \pm 0.2 \mathrm{pA} / \mathrm{mV}, n=8$ cells; $P<0.05 ;$ Supplemental Table 1$)$. In addition, the kinetics of $\mathrm{Ca}^{2+}$ current activation at negative membrane potentials were slower in both Clrn1 1ext-r $($ Figure $4 \mathrm{H})$ and Clrn $1^{e x t / f / l} \mathrm{Myo15}-\mathrm{Cre}^{+/-}$(Figure 4I) mice than in control mice, from P9 onward. Furthermore, the time-dependent inactivation of $\mathrm{Ca}^{2+}$ currents in the IHCs of the 2 clarin-1-deficient mouse mutants was greater than that in the control mice on P13 and P18 (Figure 4J), probably reflecting extended $\mathrm{Ca}^{2+}$-dependent inactivation due to a greater influx of $\mathrm{Ca}^{2+}$ (Figure 4, B, D, and E).

Overall, the Clrn1 knockout mice (Clrn $\left.1^{e x t-/}\right)$ and the postnatal hair cell-specific conditional knockout mice (Clrn1 $1^{\text {extff/fl }}$
Myo15- $\left.\mathrm{Cre}^{+/}\right)$displayed similar sequential changes in the activity and expression of IHC $\mathrm{Ca}^{2+}$ currents: a normal initial upregulation peaking on $\mathrm{P}$, followed by an abnormally weak downregulation of the density of these currents after P9. We then investigated $\mathrm{Ca}^{2+}$-triggered exocytosis in Clrnt ${ }^{\mathrm{ext}-1-}$ and $\mathrm{Clrn} 1^{\mathrm{extff} / \mathrm{fl}}$ Myo15-Cre ${ }^{+/}$IHCs (Figure 5). Analyses of the membrane capacitance changes $\left(\Delta \mathrm{C}_{\mathrm{m}}\right)$ of these IHCs in response to brief depolarizing voltage steps showed that the $\mathrm{Ca}^{2+}$ efficiency and kinetics of exocytosis were normal at P9 (Figure 5, A and C), but decreased from P13 onward (Figure 5, B and D, and Supplemental Table 2). We then probed the intracellular $\mathrm{Ca}^{2+}$ sensitivity of the exo- 
A

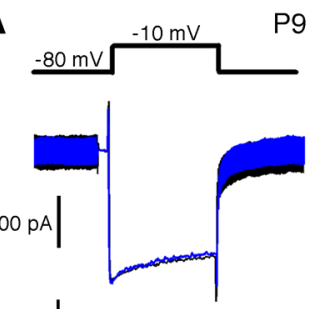
$20 \mathrm{fF} \frac{\mathrm{Ls}}{50 \mathrm{~ms}}$

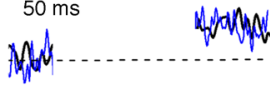

Control mice $(\bullet, 0)$

C
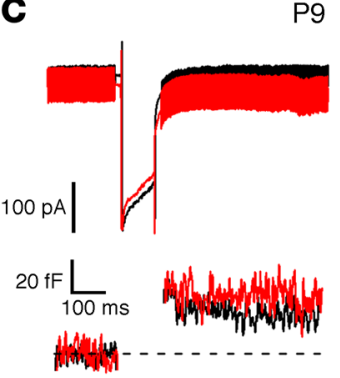

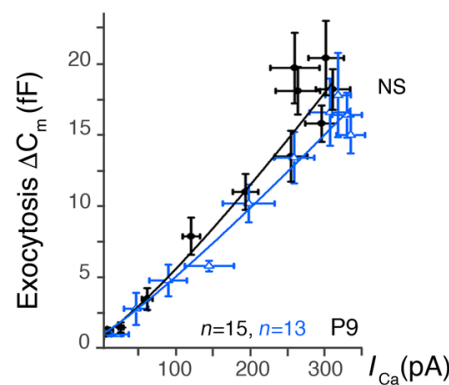

B

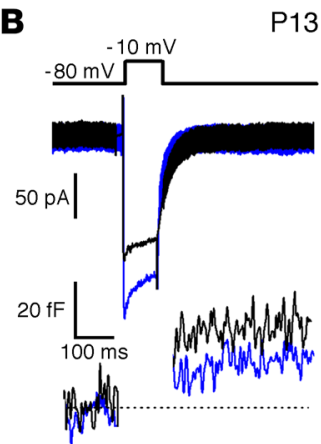

Clrn $1^{\text {ex4-/- mice }}(\Delta)$

Clrn1 1ex4iflel Myo15-Cre ${ }^{+/-}$mice

$(\square, \boldsymbol{\square})$
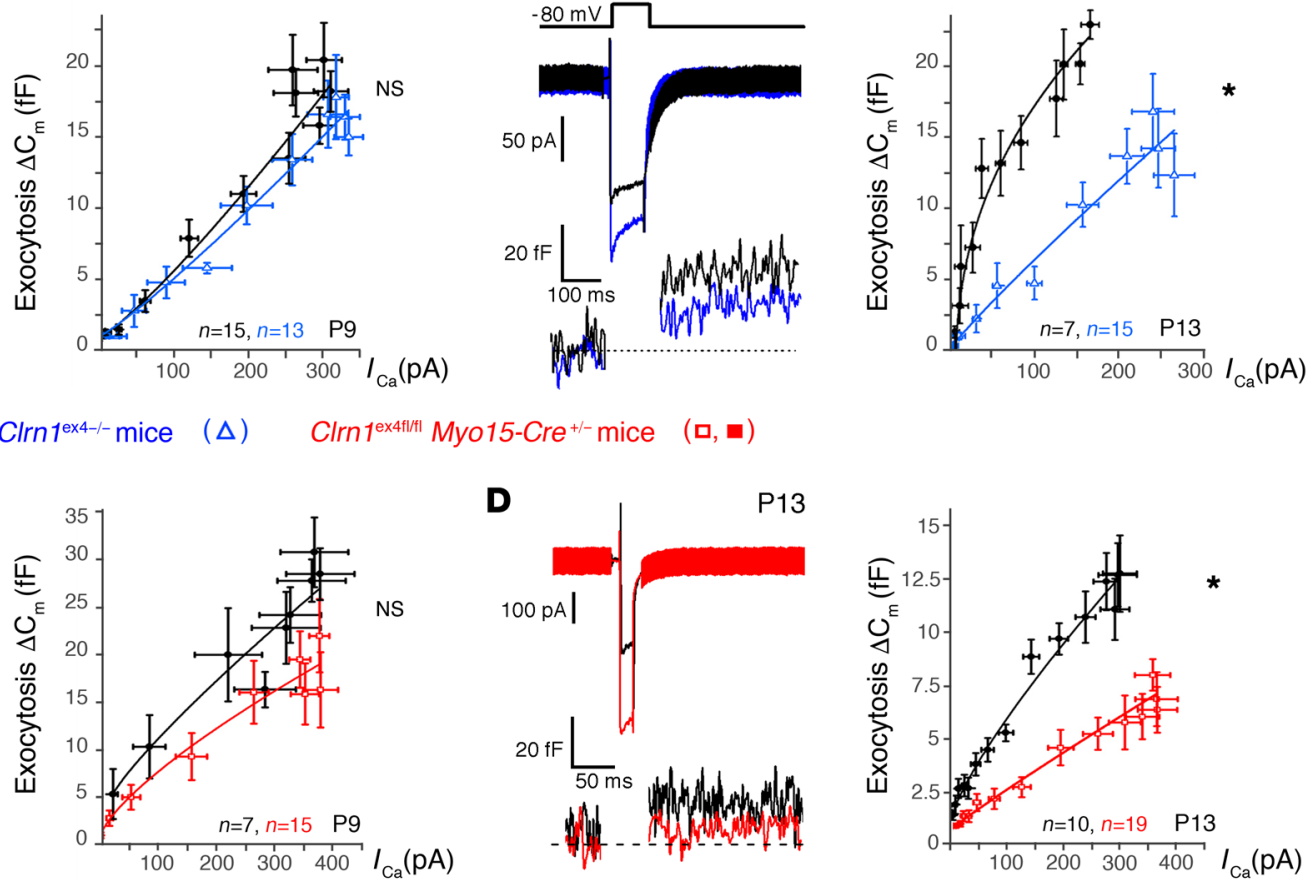

Exocytosis evoked by intracellular UV photorelease of caged-Ca ${ }^{2+}$
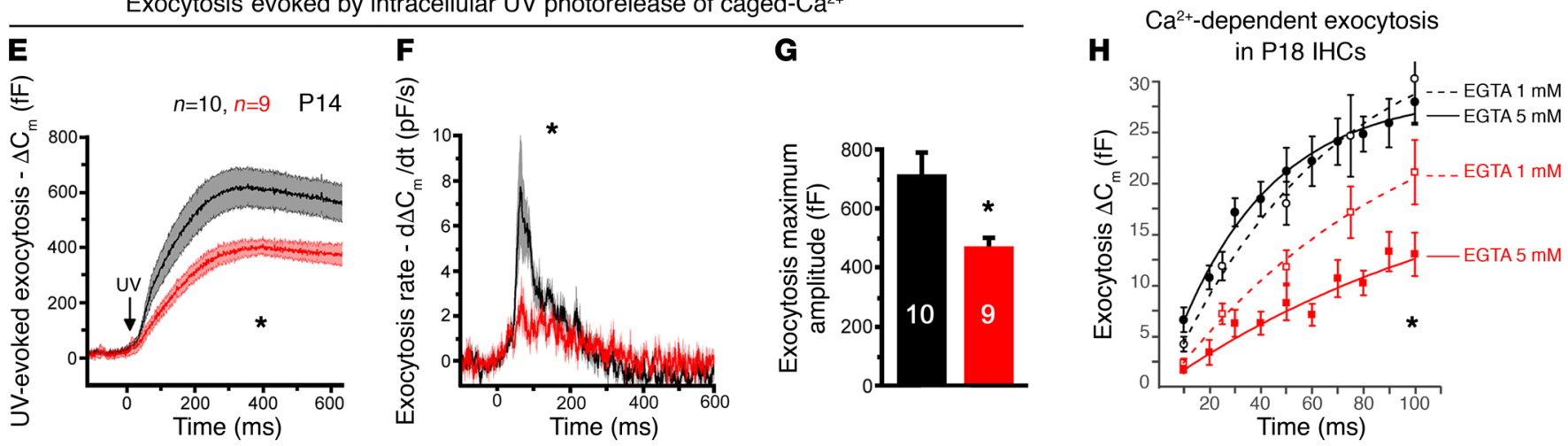

Figure 5. Ca ${ }^{2+}$-mediated exocytosis in mutant IHCs lacking clarin-1. (A-D) Exocytosis was measured after various voltage steps between -80 mV and $-5 \mathrm{mV}$, with each voltage step lasting 100 milliseconds (A-C) or 25 milliseconds (D). A simple power function was fitted to the data ( $y=a x^{N}$, where $a$ is exocytotic slope efficiency and $N$ the power index; Supplemental Table 2). Ca ${ }^{2+}$ efficiency for IHC exocytosis was normal at P9, but was significantly lower

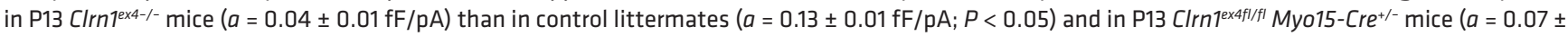
$0.01 \mathrm{fF} / \mathrm{pA}$ ) than in control littermates $(a=0.16 \pm 0.01 \mathrm{fF} / \mathrm{pA} ; P<0.05)$. (E-G) Mean exocytotic response curves evoked by UV uncaging of intracellular Ca ${ }^{2+}$ in IHCs from P14 Clrn 1exffl/fl Myo15-Cre/- (red) and control (black) mice. (F) Exocytosis rates obtained by a derivative function (dC $\mathrm{m}_{\mathrm{m}} / \mathrm{dt}$ ) of the curves shown in E. (G) Comparative exocytotic peak upon $\mathrm{Ca}^{2+}$ photorelease in mutant and control IHCs. (H) Exocytosis kinetics for constant voltage steps from -80 to $-10 \mathrm{mV}$, with durations increasing from 10 to 100 milliseconds, in IHCs from P18 Clrn pexfl/fl Myo15-Cre ${ }^{+/-}$(red) and control (black) mice. The intracellular Ca ${ }^{2+}$ buffer was $1 \mathrm{mM}$ or $5 \mathrm{mM}$ EGTA. A simple exponential function was fitted to the data, with similar time constants of $25 \pm 10$ milliseconds ( $n=12$ ) and 33 \pm 18 milliseconds $(n=11)$ for controls in $1 \mathrm{mM}$ and $5 \mathrm{mM}$ EGTA, respectively. For Clrn1 $7^{\text {exffl/fl }}$ Myo15-Cre ${ }^{+/-}$IHCs, the time constant increased from $102 \pm 50$ milliseconds $(n=27)$ in 1 mM EGTA to $205 \pm 32$ milliseconds $(n=10)$ in 5 mM EGTA $(P<0.05)$. The values shown are means \pm SEM; ${ }^{*} P<0.05(\mathbf{A}-\mathbf{F}$ and $\mathbf{H}$, 2-way ANOVA; and G, Mann-Whitney test).

cytotic release machinery in IHCs, independently of the voltage activation of the $\mathrm{Ca}^{2+}$ channels, by uncaging intracellular $\mathrm{Ca}^{2+}$ with UV flash photolysis $(19,23)$ (Figure $5, \mathrm{E}-\mathrm{G}$ ). Following $\mathrm{Ca}^{2+}$ uncaging, the IHCs of P14 Clrn 1exfl/fl Myo15-Cre ${ }^{+/}$mice had lower rates of exocytosis $\left(\mathrm{dC}_{\mathrm{m}} / \mathrm{dt}\right.$ ) (Figure $5, \mathrm{E}$ and $\mathrm{F}$ ) and lower maximum amplitudes than control IHCs (Figure $5 G$ ).

Our findings thus indicated that the absence of clarin- 1 results in an IHC synaptopathy characterized by (a) a much weaker downregulation of $\mathrm{Ca}^{2+}$ currents than normally observed at the onset of hearing, (b) a hyperpolarized voltage-activation curve and an extended calcium-dependent inactivation of $\mathrm{Ca}^{2+}$ currents, and (c) an intrinsic defect of the exocytotic machinery, as suggested by the $\mathrm{Ca}^{2+}$ uncaging experiments.

Clarin-1 is essential for the tight clustering of $C a_{v} 1.3$ channels at the IHC ribbon synapse. Given the abnormally large $\mathrm{Ca}^{2+}$ currents and the low $\mathrm{Ca}^{2+}$ efficiency of exocytosis in mutant mice after 
Number of synaptic ribbons in control and clarin-1-deficient IHCs

A Otoferlin and ribeye immunostaining in $\mathrm{HCs}$

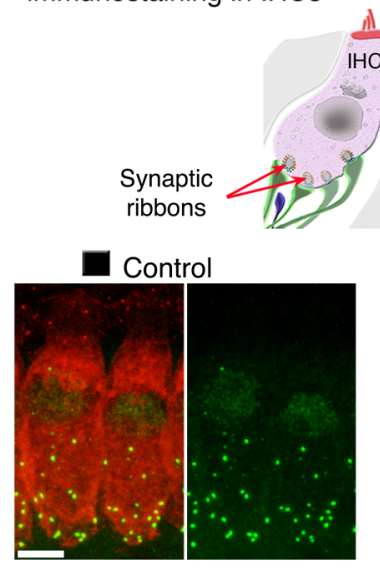

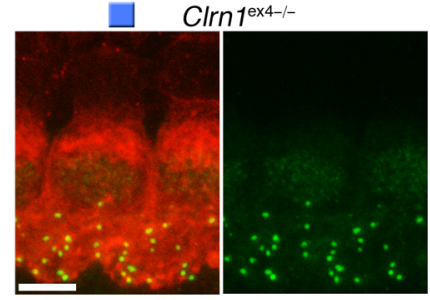

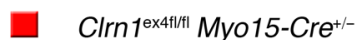

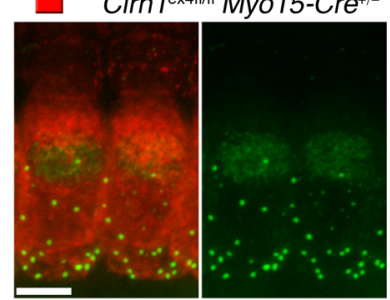

B Number of synaptic ribbons per IHC

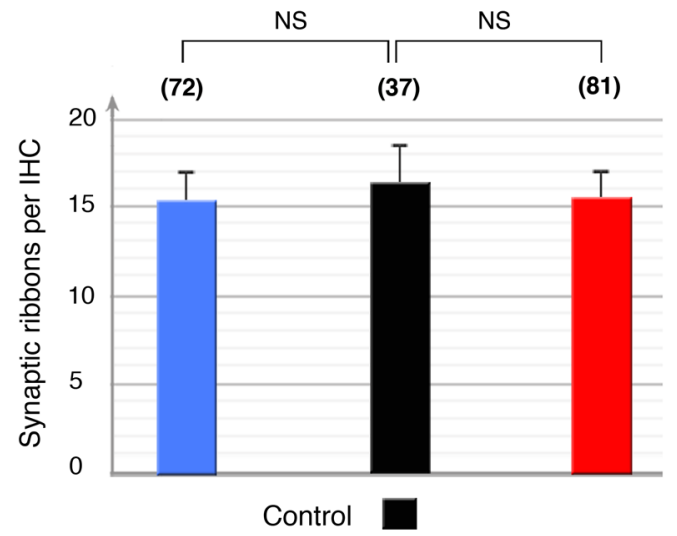

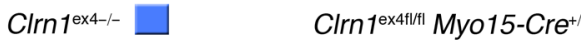

Abnormal efferent synaptic connection in clarin-1-deficient mice

C Afferent and efferent synaptic connection in a control IHC

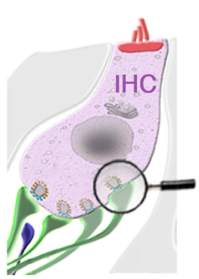

Afferent terminals

Efferent terminals

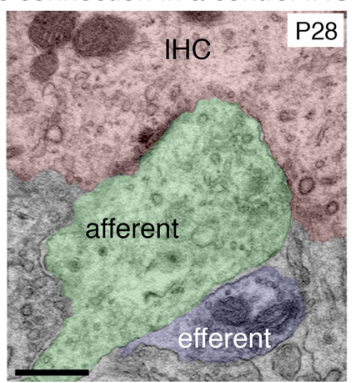

D

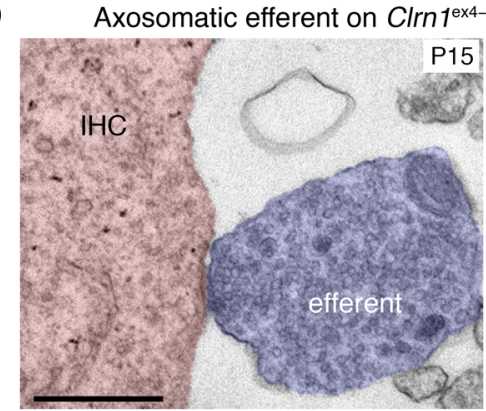

P15 and P28 IHCs

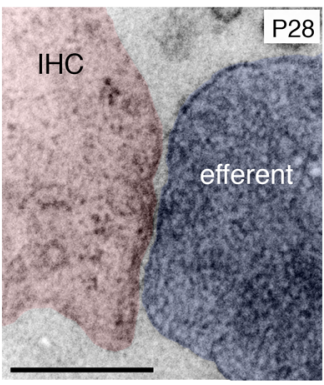

Synaptic ribbons in control and clarin-1-deficient mice

E Morphology of the IHC synaptic ribbons

Control mice

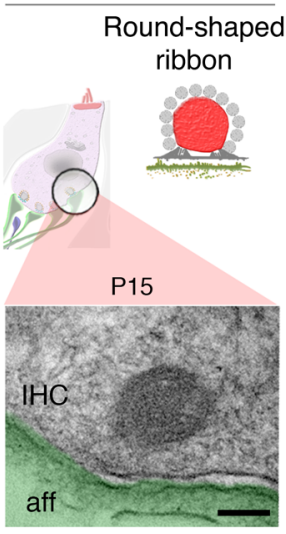

Clrn1 $1^{\mathrm{ex} 4-/-}$ mice

P15

Droplet-shaped

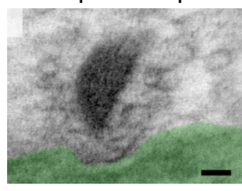

P15

Round-shaped

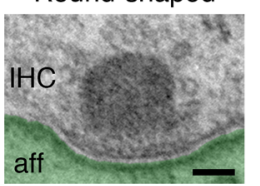

Oval-shaped

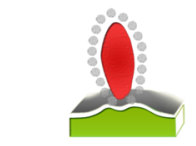

P28

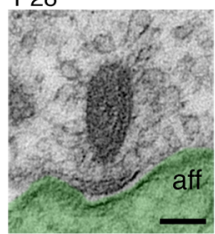

F Percentage of ribbons with mature and immature shape

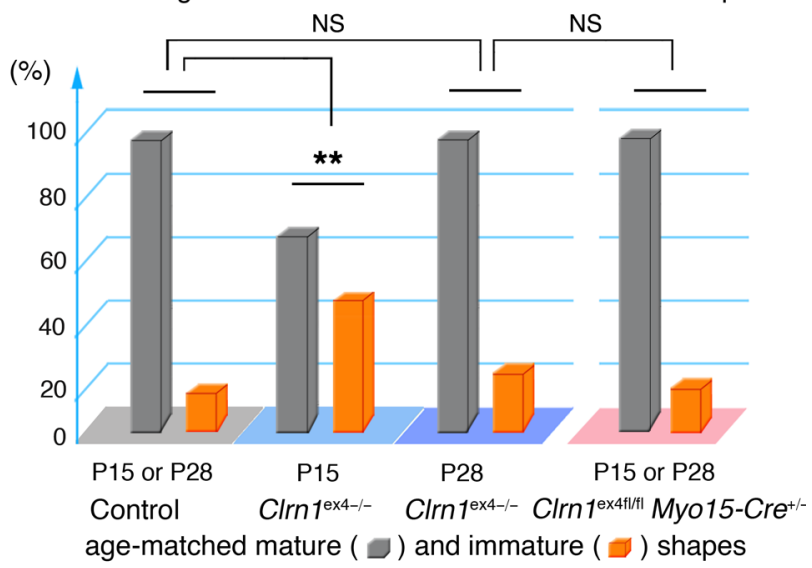

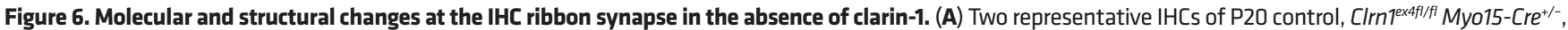
and $\mathrm{Cl}_{\mathrm{rn}} \mathrm{7}^{\text {pxu- }}$ mice stained for otoferlin (red) and ribeye (green), showing the correct location of the ribbons in the basolateral region of mutant IHCs despite the absence of clarin-1. (B) The bar chart shows the number (mean \pm SEM; unpaired Student's $t$ test) of synaptic ribbons per IHC, which is similar in P20 Clrn 7ex4-/- (blue), Clrnn fexfflffl Myo15-Cre ${ }^{+/-}$(red), and control (black) mice. (C) In control IHCs, efferent terminals (artificially colored in light blue) contact only afferent terminals (green). (D) By contrast, in Clrn 1ex4-/- mice, efferent nerve fibers (blue) are found in direct contact with IHCs, on both P15 and P28. (E) Round, oval, and droplet-shaped ribbons are present in mutant IHCs lacking clarin-1 on P15. Each transmission electron microscopic image in C-E is representative of 5-10 IHCs analyzed from 3 different mice

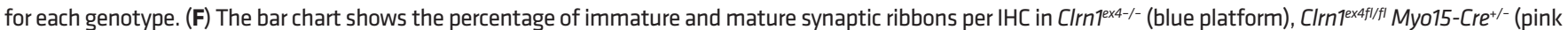
platform), and control (gray platform) mice. The IHCs of Clrn 7exffl/fl Myo15-Cre $\mathrm{e}^{+/-}$mice have normal frequencies of round (immature) and droplet-shaped (mature) ribbons on P15 (E and F). Similar values were obtained on P15 and P28. In the IHCs of Clrn 7ex4-/- mice, round ribbons were numerous on P15, but their frequency had decreased to normal values on P28 (F). ${ }^{* *} P<0.01$ (Mann-Whitney test). Scale bars: $5 \mu \mathrm{m}(\mathbf{A}), 100 \mathrm{~nm}(\mathbf{C}, \mathbf{D}$, and E). 
A $\mathrm{Ca}_{\mathrm{v}}$ 1.3-immunoreactive patches per $\mathrm{IHC}$

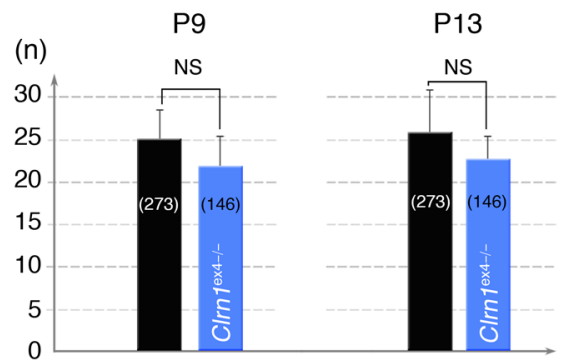

C Fraction of $\mathrm{Ca}_{\mathrm{v}} 1$.3-immunoreactive area also labeled for ribeye (\% per patch)

P9

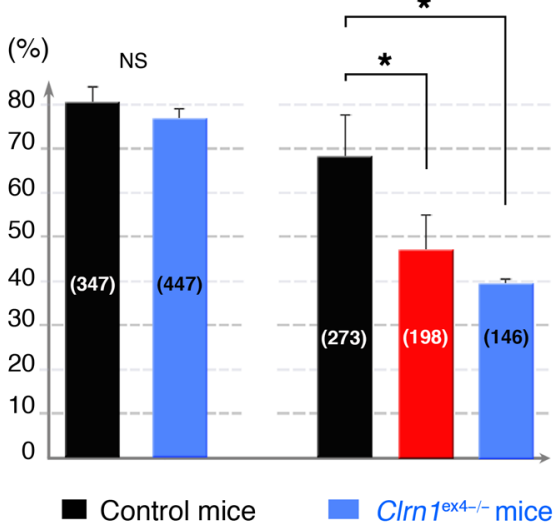

Clrn1ex4l/ili Myo15-Cre + - mice
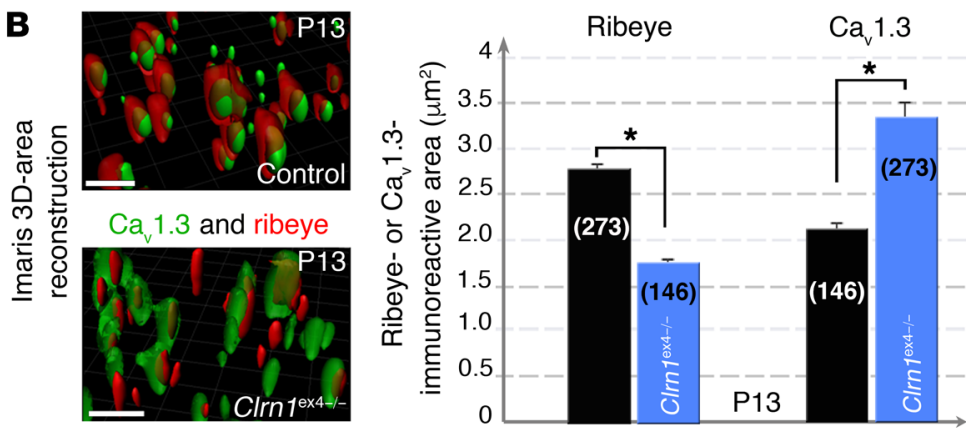

D

$\mathrm{Ca}_{\mathrm{v}}$ 1.3- and ribeye-labeled patches in IHCs of control, Clrn $1^{\mathrm{ex} 4-\mathrm{-}}$, and Clrn1 ${ }^{\text {exAll/fl }}$ Myo15-Cre ${ }^{+/-}$mice

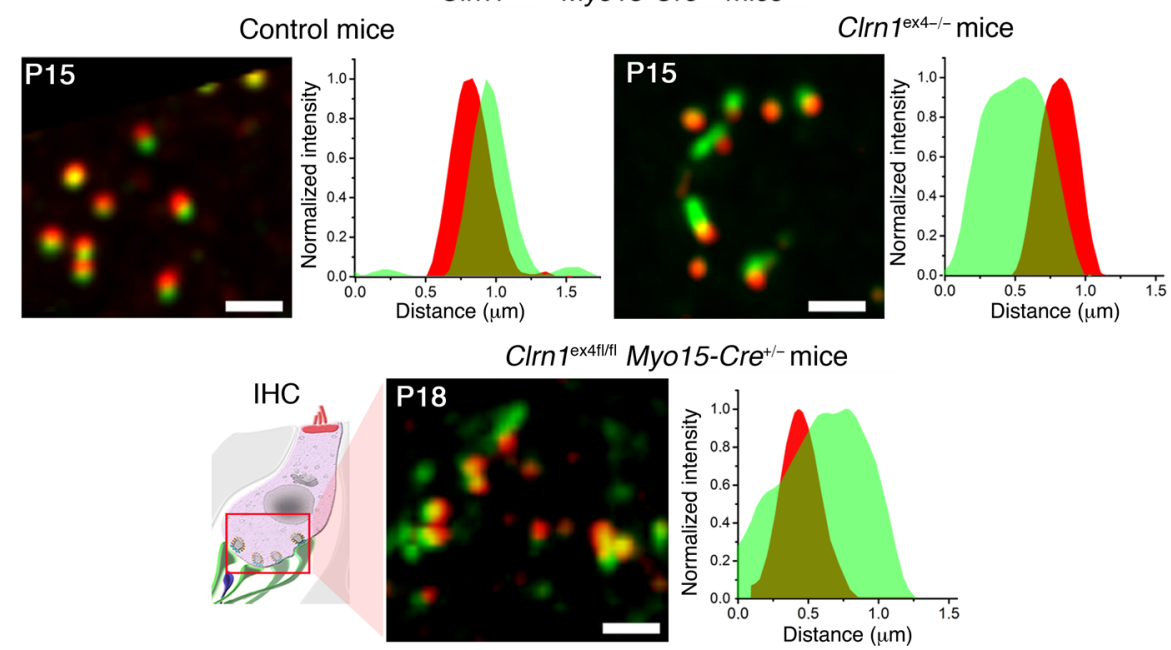

F F-actin, ribeye, and $\mathrm{Ca}_{v} 1.3$ in IHCs of $\mathrm{P} 13$ mice

E F-actin, ribeye, and $\mathrm{Ca}_{\mathrm{v}} 1.3$ in IHCs of P9 mice
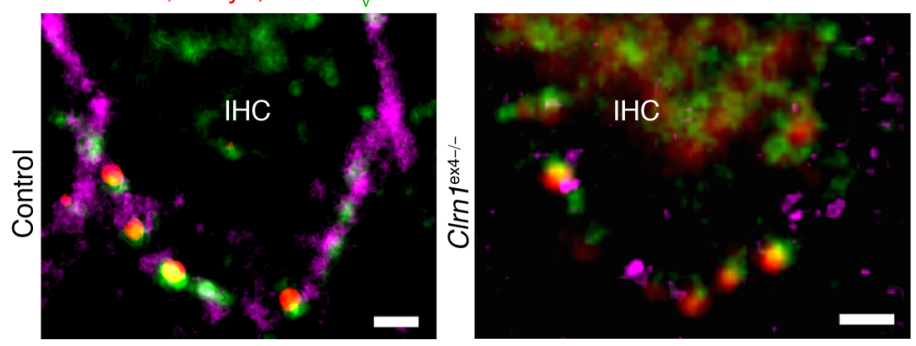

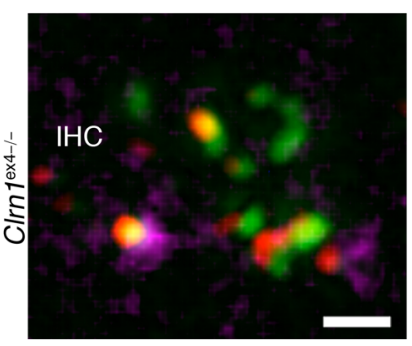

Figure 7. Confocal imaging of $\mathrm{Ca}_{\mathrm{v}} \mathbf{1 . 3}$ channels and F-actin at IHC ribbon synapses lacking clarin-1. (A) The number of Ca 1.3 -immunoreactive patches associated with ribbons per IHC is similar in control (black) and Clrn7 7x4-/- (blue) mice, on both P9 and P13. The numbers of Ca 1.3 patches counted are indicated in parentheses. (B) Representative Imaris 3D images and quantitative analysis (bar chart) of immunoreactive patches in the synaptic basal region of IHCs from P13 control (black) and Clrn1 ${ }^{\text {ex4-/- }}$ (blue) mice. Ca 1.3 channels form larger patches in the active zone, and the ribbons are smaller in IHCs lacking clarin-1 than in control IHCs. (C) The rate of colocalization for $\mathrm{Ca}_{\mathrm{v}} 1.3$ - and ribeye-immunoreactive areas is normal on P9, but low on P13 in the absence of clarin-1. (D) Representative confocal images of Ca 1.3 -immunoreactive (green) and ribeye-immunoreactive (red) patches, and a

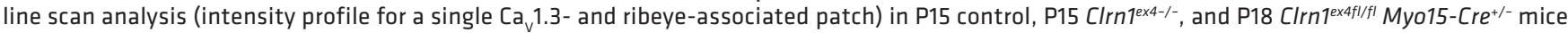
(images representative of 6 mice for each genotype). (E and $\mathbf{F}$ ) Cytoskeleton architecture in IHCs from control and Clrn ${ }^{\text {ex4- } /-}$ mice. The F-actin cortical network (purple) is altered in the IHC synapse region of CIrn ${ }^{\text {px4-/- }}$ on P9 $(\mathbf{E})$ and P13 $(\mathbf{F})$. The bar charts show the mean values \pm SEM; ${ }^{*} P<0.05$ (unpaired Student's $t$ test). Scale bars: $1 \mu \mathrm{m}$.

P9, we investigated possible defects of the spatial distribution and architecture of the synaptic active zones of IHCs. On P21, the mean number of ribbons per IHC in Clrn1 $1^{\text {ext-/- }}$ and Clrn1 $1^{\text {ex } 4 f / f l}$ Myo15-Cre ${ }^{+/-}$mice was similar to that in age-matched control IHCs (mean \pm SEM and number of IHCs for the different genotypes:

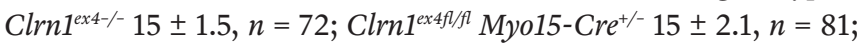
and control mice $16 \pm 2.2, n=37$ ); ribbons were counted in the
IHCs of the apical mid-turn of the cochlea (encoding frequencies of 8-16 kHz), in which exocytosis was studied (Figure 6, A and B). On ultrastructural analysis, the IHCs of P15 Clrn $1^{\text {ex4-/- }}$ mice were found to have features typical of immature IHCs, including a large fraction of spherical ribbons and the persistence of axosomatic efferent synaptic contacts (Figure 6, C and D). Only 60\% of the ribbons (19 of the 31 ribbons examined from $3 \mathrm{Clrn}^{\text {ext-/- }}$ mutant 
A

AAV2/8-CIrn1-IRES-GFP

ITR CAG mClrn1 IRES GFP ITR

Clrn 1 $1^{\mathrm{x} 4-\mathrm{-}}$ IHCs expressing the Clrn1 cDNA

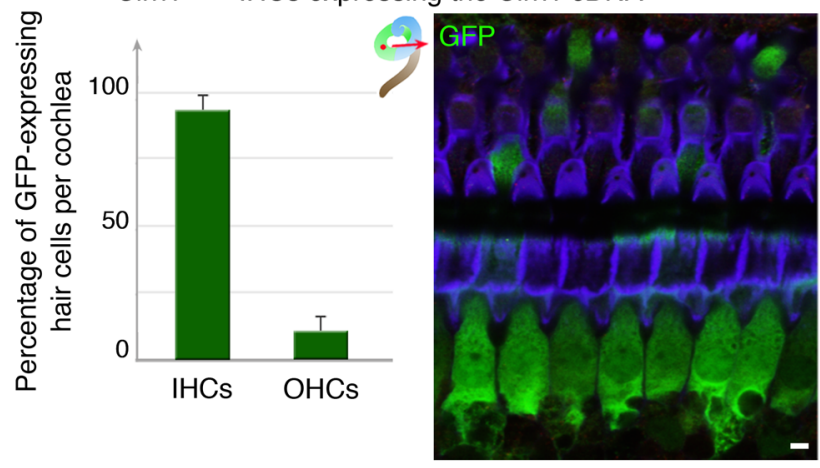

B $\quad$ ABR thresholds in mice with or without viral injections of either AAV2/8-CIrn1-IRES-GFP or AAV2/8-GFP alone

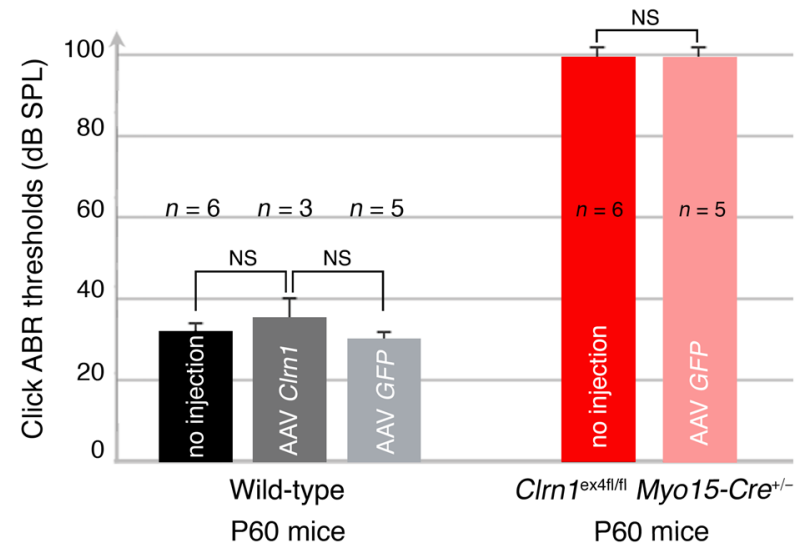

C Hearing upon viral injections in P22-P24 Clrn $1^{\text {ex4-/-mice }}$

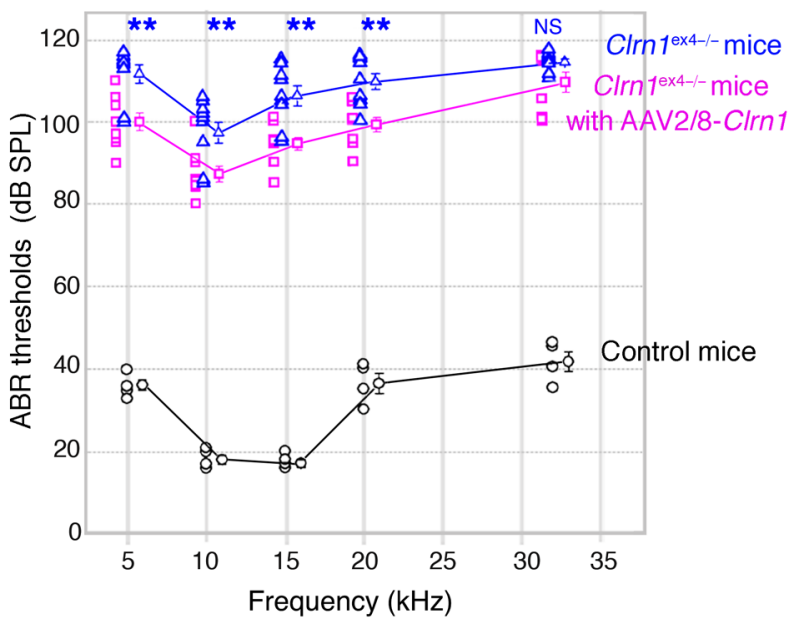

D
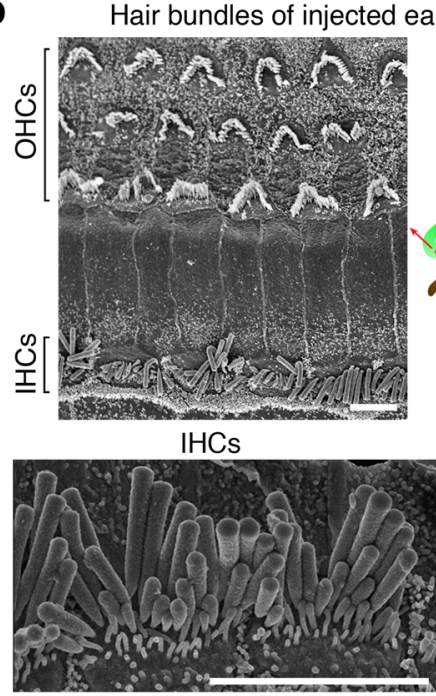

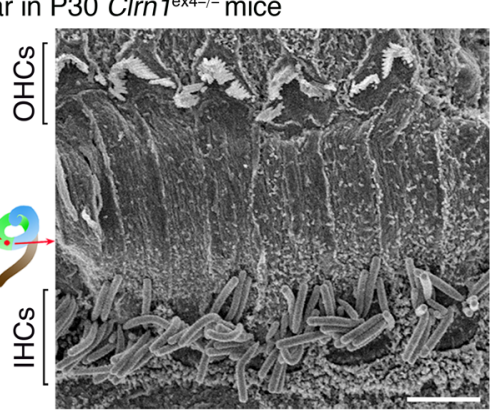

$\mathrm{OHCs}$

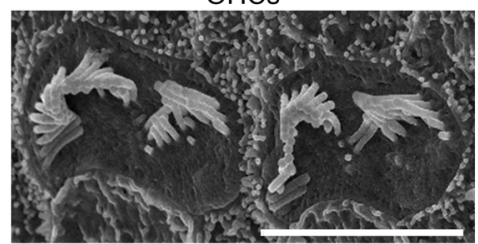

Figure 8. Intracochlear AAV2/8-mediated delivery of clarin-1 in Clrn 1ex4-/- mice leads to moderate hearing preservation. (A) The schematic diagram (top panel) shows the AAV2/8-CIrn1-IRES-GFP vector used for gene delivery to the cochlea on P2-P3. The histogram indicates the percentage of GFP-labeled IHCs and OHCs on P20-P30 in Clrn7 7x4-/- mice. (B) Injections of either AAV2/8-Clrn1-IRES-GFP or AAV2/8-GFP had no effect on click ABR thresholds in WT mice. Injections of AAV2/8-GFP also had no impact on ABR thresholds in Clrn fexafl/fl Myo15-Cre ${ }^{+/-}$mice (unpaired Student's $t$ test). (C) ABR thresholds (mean \pm SEM) in treated (purple, $n=9$ ) and untreated (blue, $n=9$ ) inner ears of P22-P24 Clrn pex4-/ and control (black, $n=5$ ) mice. A decrease of about 10-15 dB in ABR thresholds relative to untreated ears was observed in Clrn $7^{\text {ex4-/- }}$ ears after injection. ${ }^{* *} P<0.01$ (unpaired Student's $t$ test). (D) Early postnatal AAV2/8-mediated delivery of clarin-1 into Clrn7ex4-/- ears did not prevent or correct the misshaping of the hair bundles. Both IHC and OHC hair bundles presented alterations in shape and a loss of short-row stereocilia due to the embryonic loss of clarin-1 (representative of 8 mice between P25 and P30). Scale bars: $1 \mu \mathrm{m}$.

mice) had a mature plate-like shape similar to that observed in control mice of the same age (Figure 6, E and F). Over subsequent days, the fraction of immature ribbons observed in Clrn1 $1^{\text {ex4- }-1}$ IHCs decreased from $40 \%(n=31)$ on P15 to less than $20 \%(n=17)$ on P28, thereby reaching near-normal values (Figure 6F). The shapes of the synaptic ribbons in Clrn1 $1^{\text {ex } 4 f / f l} \mathrm{Myo15}-\mathrm{Cre}^{+/-} \mathrm{IHCs}$ were indistinguishable from those in control IHCs, on both P15 and P28. On P28, most of the ribbons ( $93 \%, n=42$ ) had the plate-like shape typical of mature synapses (Figure 6F). Thus, the early constitutive deletion of clarin-1 in Clrn $1^{\text {ext-/ }}$ IHCs delayed the morphological maturation of the synaptic ribbons.

$\mathrm{Ca}^{2+}$-mediated exocytosis was less efficient in the absence of clarin-1. We therefore characterized the coupling of $\mathrm{Ca}^{2+}$ influx and exocytosis, by probing exocytosis following the application of vari- ous intracellular concentrations of the $\mathrm{Ca}^{2+}$ chelator EGTA (Figure $5 \mathrm{H})$. After hearing onset, the $\mathrm{Ca}^{2+}$ channels and synaptic vesicles of normal mouse IHCs are spatially organized into a nanodomain configuration, which is thought to account for the lack of sensitivity of exocytosis to EGTA in these cells (24). Accordingly, increasing the concentration of intracellular EGTA from 1 to $5 \mathrm{mM}$ did not affect the kinetics of exocytosis evoked during brief pulses, from 10 to 100 milliseconds, in P18 control mice ( $\tau=25 \pm 10 \mathrm{~ms}$ and 33 $\pm 18 \mathrm{~ms}$, respectively, $P=0.5$; Figure $5 \mathrm{H}$ ). This time is generally thought to correspond to the time required for the release of the readily releasable pool of vesicles in IHCs at room temperature $\left(22^{\circ} \mathrm{C}-24^{\circ} \mathrm{C}\right)$ and with $5 \mathrm{mM}$ extracellular $\mathrm{Ca}^{2+}(24,25)$. By contrast, the incubation of $5 \mathrm{mM}$ intracellular EGTA with P18 IHCs from Clrn $1^{e x 4 f / f l} \mathrm{Myo15-Cre^{+/- }}$ mice led to a further decrease in the 
A

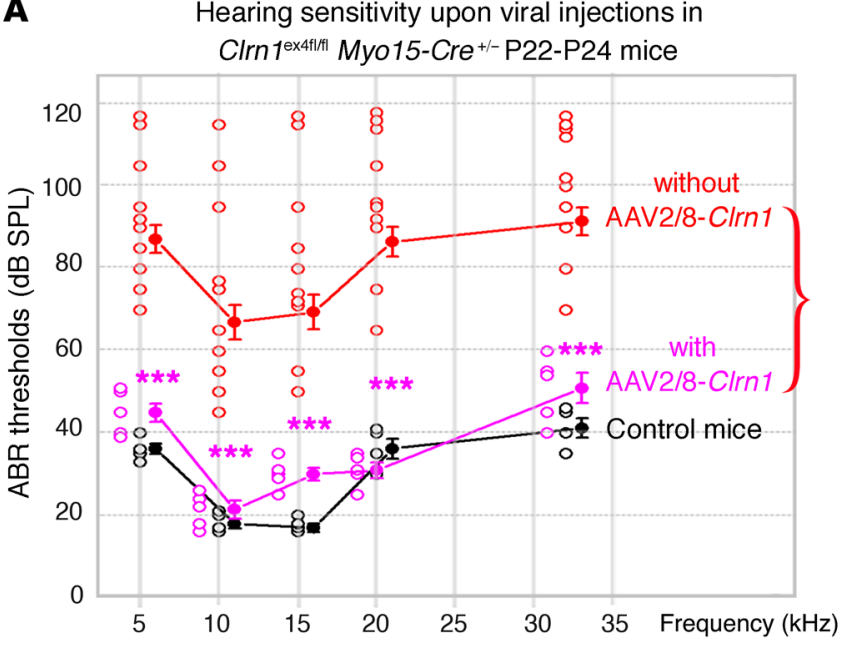

Control mice $(\bullet, 0)$
B Hearing sensitivity upon viral injections in

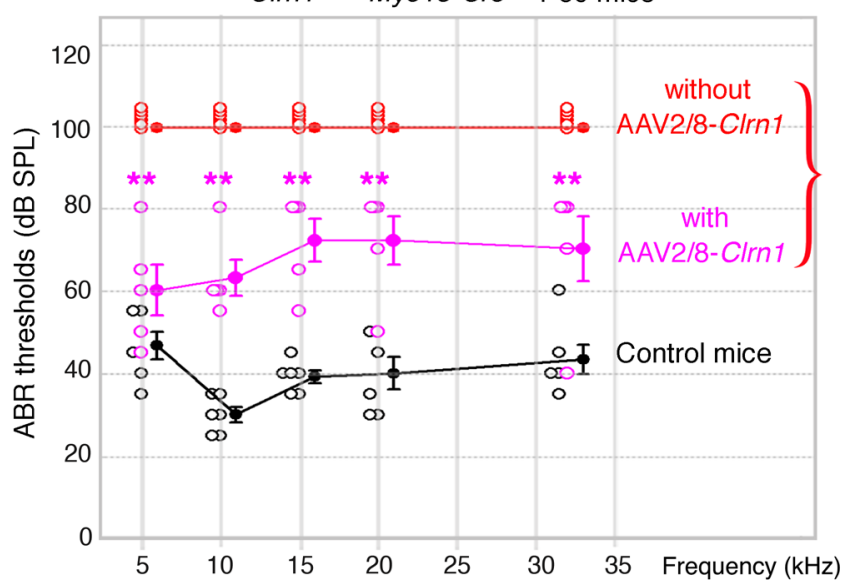

Clrn1 1ex4l/1/1 Myo15-Cre ${ }^{+/-}$mice with AAV2/8-CIrn1 $(\bullet, 0)$
C Clrn $1^{\text {extitit }}$ control mouse (P60)

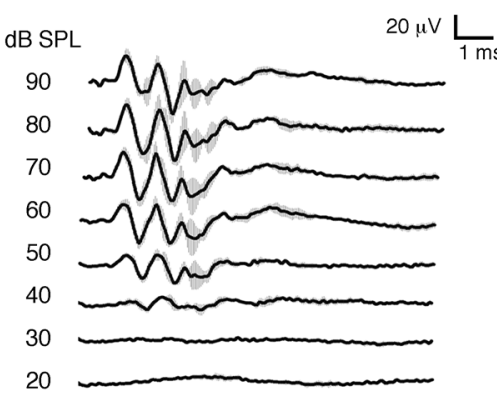

Clrn1 ex4f|lif $^{\text {Myo15-Cre }}{ }^{+/-}$mouse (P60)

dB SPL $\quad 5 \mu \mathrm{V} \underset{1 \mathrm{~ms}}{\mathrm{~L}}$

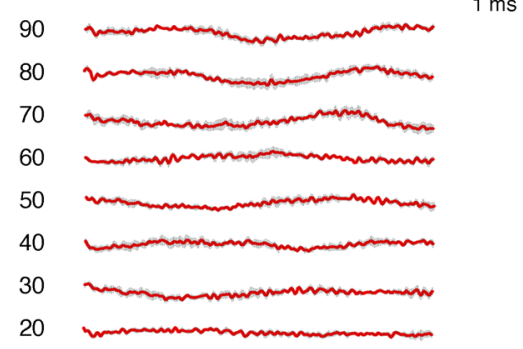

Clrntex4l/lfl Myo15-Cre+/- mouse (P60) dB SPL with AAV2/8-Clrn1 $5 \mu \vee \frac{\text { L }}{1 \mathrm{~ms}}$

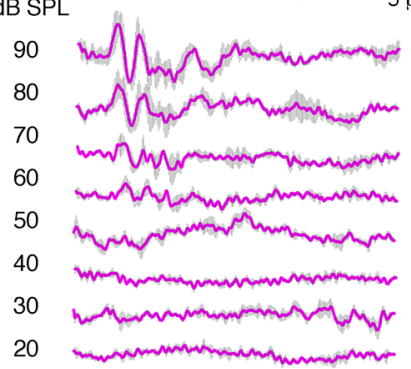

D The morphology of auditory hair bundles in Clrn 1ex4//f/l Myo15-Cre ${ }^{+/-} \mathrm{P} 120$ mice

The hearing organ of a noninjected ear in a

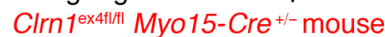

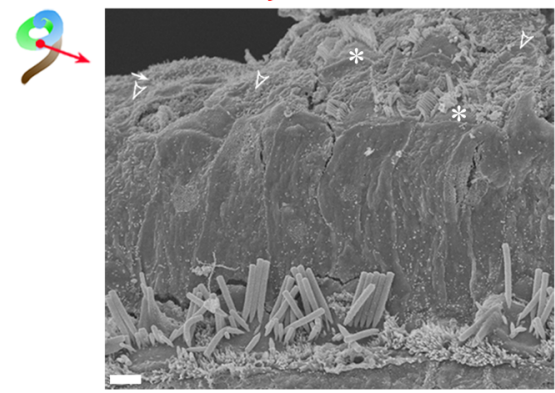

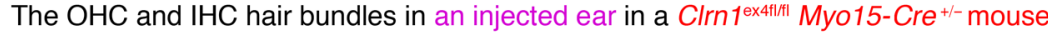

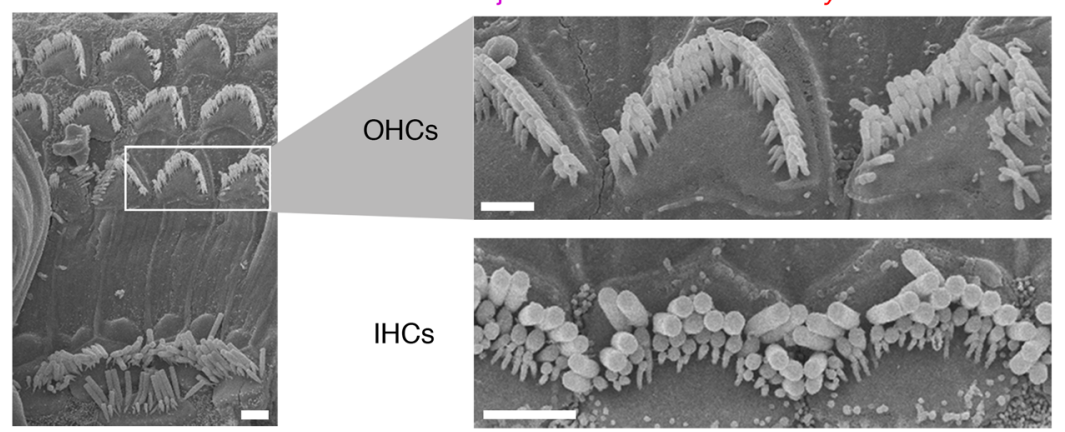

Figure 9. AAV2/8-mediated delivery of clarin-1 in Clrn 1exafl/f1 Myo15-Cre+/- mice leads to long-term preservation of hearing. (A) Comparative tone-burst ABR thresholds (audiogram, mean \pm SEM and individual points for each frequency) in AAV2/8-Clrn1-injected (purple, $n=7$ ) and untreated (red, $n=10$ ) inner ears of P22-P24 Clrn7 ${ }^{\text {exafl/fl }}$ Myo15-Cre ${ }^{+/-}$mice and control (black, $n=6$ ) mice. (B) Similar comparative audiograms for AAV2/8-Clrn1-injected (purple, $n$ $=5$ ) and untreated (red, $n=6$ ) inner ears of P60 Clrn 1ex4fl/fl Myo15-Cre+/- mice and P60 control (black, $n=6$ ) mice. (C) Mean click ABR waves at P60 in control and in untreated and AAV2/8-CIrn1-injected Clrn 1exafl/fl $M y 015-C r e^{+/-}$mice. (D) Scanning electron microscopy view of stereocilia from untreated (left) and AAV2/8-Clrn1-injected (right) ears of P120 Clrn1 ${ }^{\text {exffl/fl }} \mathrm{Myo15}^{-C \mathrm{Cr}^{+/-}}$mice $(n=3)$. By contrast to the untreated ears, the injection of AAV2/8-Clrn1 resulted in apparently normal hair bundles still visible on P120 $(n=3)$. However, despite the persistence of V-shaped bundles, the stereocilia in the short and middle rows are heterogeneous in length, and many of these stereocilia are missing. ${ }^{* *} P<0.01,{ }^{* *} P<0.001$ (unpaired Student's $t$ test). Scale bars: $1 \mu \mathrm{m}$.

rate of exocytosis, which was already slow in the presence of $1 \mathrm{mM}$ EGTA (Figure $5 \mathrm{H}$ ). This sensitivity to internal EGTA concentration suggests that $\mathrm{Ca}^{2+}$ channels are loosely coupled with the sites of synaptic vesicle release in IHCs lacking clarin-1. Immunostaining for $\mathrm{Ca}_{\mathrm{v}} 1.3$ and ribeye (a component of the ribbon) on P9 and P13 revealed similar numbers per IHC and a basolateral distribution

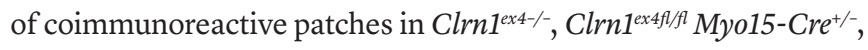
and control mice (Figure 7A). However, the mean surface area of ribeye-immunoreactive patches was much smaller in Clrn $1^{\text {ex4-/- }}$ mice than in controls on $\mathrm{P} 13$, as estimated by $3 \mathrm{D}$-surface area reconstruction with Imaris software (Bitplane AG) $\left(1.77 \pm 0.07 \mu \mathrm{m}^{2}\right.$, $n=146$ ribbons, in P13 Clrn1 $1^{\text {ex4-/- }}$ mice, vs. $2.77 \pm 0.09 \mu \mathrm{m}^{2}, n=273$ 
A Synaptic activity in IHCs of control and Clrn 1ex4-/- injected and noninjected ears

\section{- Injected ears of}

control mice $(n=9)$

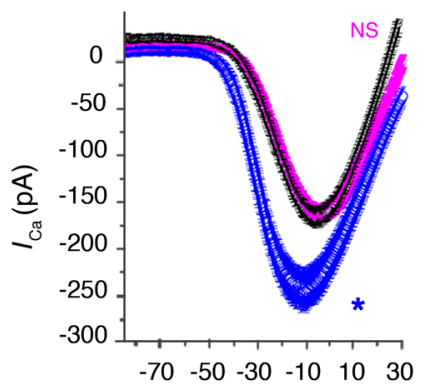

Membrane potential $(\mathrm{mV})$
- Injected ears of

Clrn $1^{\text {ex4-/- }}$ mice $(n=16)$

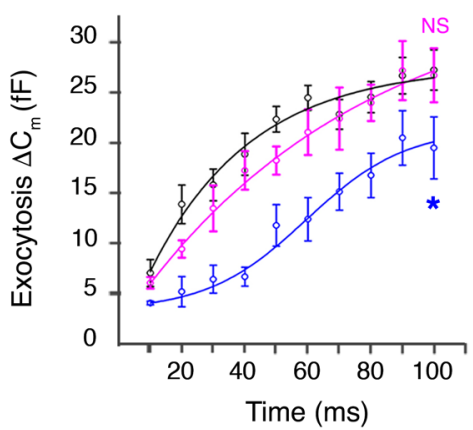

- Contralateral (noninjected) ears of $C / r n 1^{\text {ex4-/- }}$ mice $(n=9)$

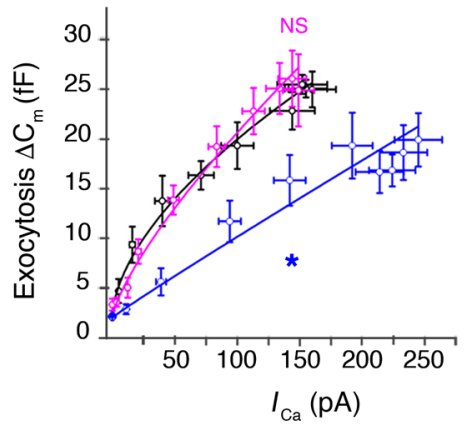

B
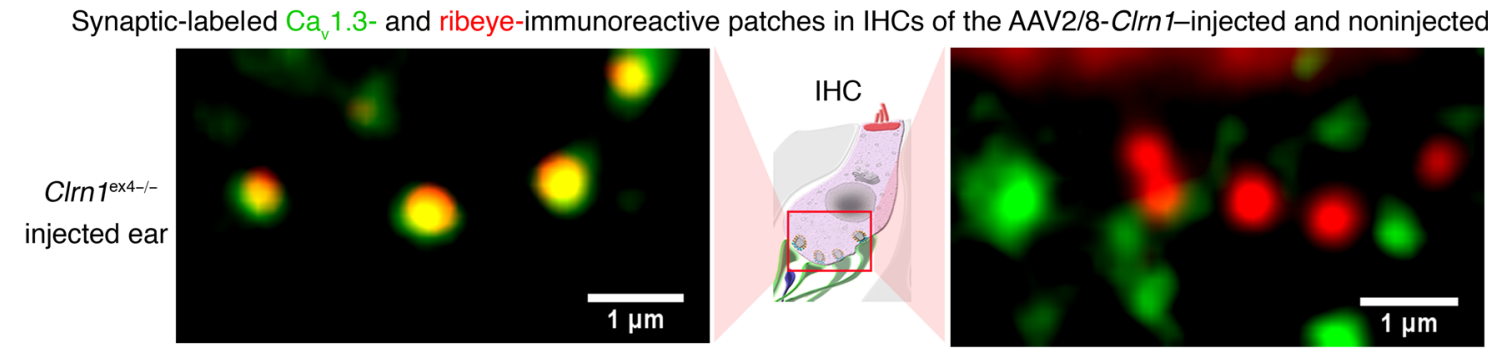

Clrn $1^{\mathrm{ex} 4-\mathrm{-}}$
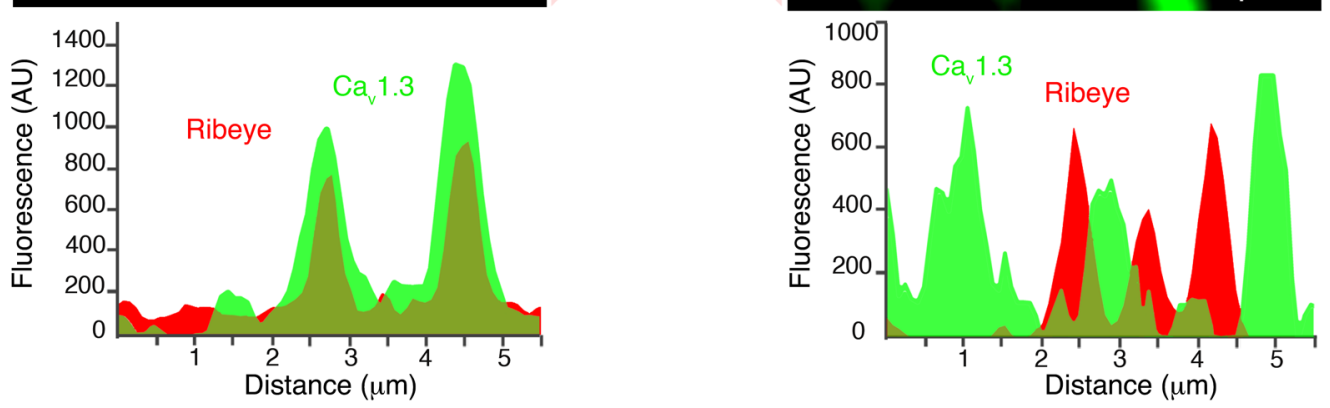

Figure 10. AAV2/8-mediated delivery of Clrn1 in Clrn 1ex4-/- mice preserves synapse ribbon structure and function. (A) The re-expression of clarin-1 at the IHC synapse (P15-P18) in Clrn7 1ex4-/ IHCs led to normal Ca ${ }^{2+}$ currents, kinetics, and Ca ${ }^{2+}$ efficiency of exocytosis (Supplemental Table 3). ${ }^{*} P<0.05$ (2-way ANOVA). (B) The virus-mediated expression of clarin-1 restored the tight clustering of $\mathrm{Ca}^{2+}$ channels in the IHC active zone, as shown by the line scan intensity profiles of $\mathrm{Ca}_{\mathrm{v}} 1.3$ - and ribeye-immunoreactive patches for 2 ribbons (images representative of 3 mice).

ribbons, in control mice; $P<0.05$; Figure 7B). Conversely, the mean size of the $\mathrm{Ca}_{\mathrm{v}} 1.3$-immunoreactive patches was almost $40 \%$ larger in P13 Clrn1 $1^{\text {ext- }-}$ mice $\left(3.33 \pm 0.17 \mu \mathrm{m}^{2}, n=146\right.$ ribbons) than in $\mathrm{P} 13$ control mice $\left(2.10 \pm 0.10 \mu \mathrm{m}^{2}, n=273\right.$ ribbons; $P<0.05$; Figure $\left.7 \mathrm{~B}\right)$. The proportion of the total area immunoreactive for both $\mathrm{Ca}_{\mathrm{v}} 1.3$ and ribeye (an index of spatial proximity) in each active zone on P9 was not significantly different in Clrn1 ${ }^{\text {ext-/- }}$, Clrn1 $1^{\text {ex4fl/fl }}$ Myo15$\mathrm{Cre}^{+/-}$, and control mice $\left(80 \% \pm 5 \%, n=447\right.$ ribbons, in Clrn1 ${ }^{\text {ex4-/- }}$ mice vs. $77 \% \pm 2 \%, n=347$ ribbons, in controls; $P=0.5$; Figure $7 \mathrm{C}$ ). By contrast, this proportion was lower in $\mathrm{Clrn1}^{\text {ex4-/- }}$ and $\mathrm{Clrn} 1^{\text {ex4ff/fl }}$ Myo15- $\mathrm{Cre}^{+/-}$mice on P13 $\left(40 \% \pm 1 \%, n=146\right.$ ribbons, in Clrn1 ${ }^{\text {ex4-- }}$ mice, and $48 \% \pm 6 \%, n=198$, in Clrn1 ${ }^{\text {ex } 4 f l / f l} \mathrm{Myo15}_{\mathrm{Cr}} \mathrm{Cr}^{+/}$mice, vs. $69 \% \pm 8 \%, n=273$, in control mice; $P<0.05$; Figure 7, C and D). In P21 Clrn1 $1^{\text {ex } 4 f / f l}$ Myo15-Cre ${ }^{+/-}$mice, this proportion was $30 \% \pm 6 \%$ ( $n=205$ ribbons) of that in control IHCs ( $n=181$ ribbons; $P<0.05)$. These results highlight a loose spatial coupling between $\mathrm{Ca}^{2+}$ channels and the synaptic machinery in the absence of clarin-1.

The synaptic F-actin network is essential for the maintenance of a tight spatial organization of $\mathrm{Ca}_{\mathrm{v}} 1.3 \mathrm{Ca}^{2+}$ channels in IHC active zones (23). In control IHCs on P9, the submembranous F-actin cortical network was intensely stained and closely associated with the ribeye- and $\mathrm{Ca}_{\mathrm{v}} 1.3$-coimmunoreactive spots (Figure $7 \mathrm{E}$ ). By $\mathrm{P} 13$, this F-actin staining had extended further beneath the control IHC plasma membrane, forming dense intracellular cylindrical meshes of $0.5^{-1} \mu \mathrm{m}$ in diameter (23). Synaptic F-actin staining was associated with 1 or 2 ribeye- and $\mathrm{Ca}_{\mathrm{v}} 1.3$-coimmunoreactive patch-

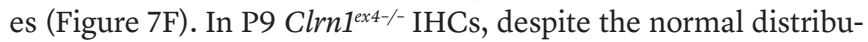
tion of ribeye and $\mathrm{Ca}_{\mathrm{v}} 1.3$, the continuous submembranous F-actin network appeared to be disrupted, as shown by the much weaker and fragmented pattern of F-actin staining (Figure 7E). On P13, synaptic F-actin staining in the IHCs of Clrn1 $1^{e x 4-/-}$ and Clrn1 $1^{e x 4 f / f l}$ Myo15-Cre ${ }^{+/-}$mice was even weaker, indicating a clear disorganization of the dense intracellular subcortical mesh network of F-actin (Figure 7F, and data not shown).

The loss of clarin-1, therefore, led to disorganization of the synaptic F-actin network in IHCs, probably accounting for the abnormally large clusters of $\mathrm{Ca}_{\mathrm{v}} 1.3 \mathrm{Ca}^{2+}$ channels in the vicinity of the synaptic ribbons. 
A Clarin-1 and $\mathrm{Ca}_{\mathrm{v}} \beta_{2}$ interaction
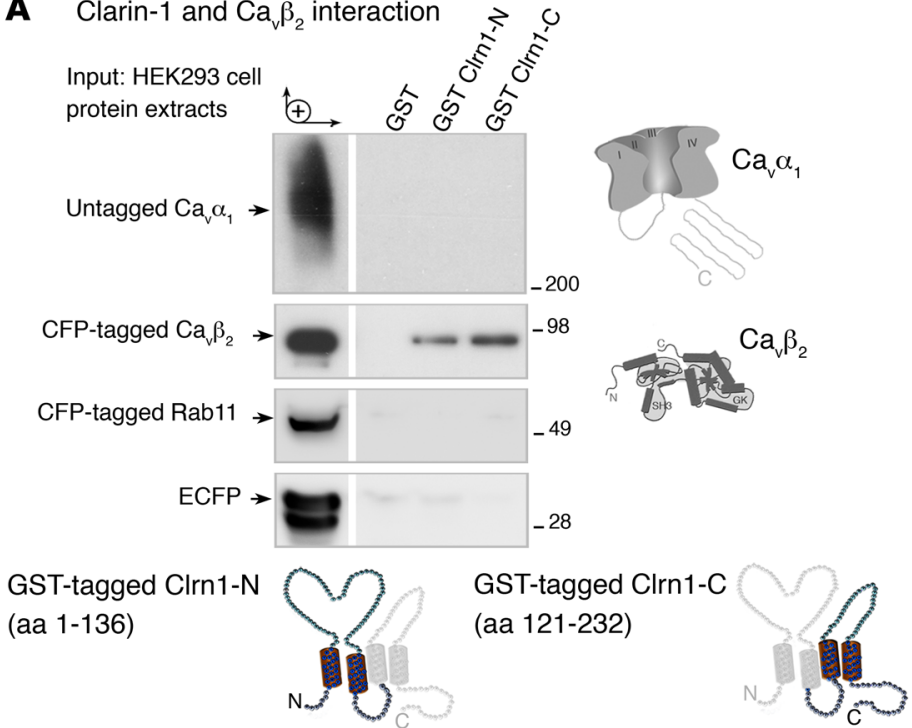

C Clarin-1, $\mathrm{Ca}_{\mathrm{v}} \beta_{2}$, and $\mathrm{Ca}_{\mathrm{v}} \alpha_{1} / \mathrm{AID}$ interaction

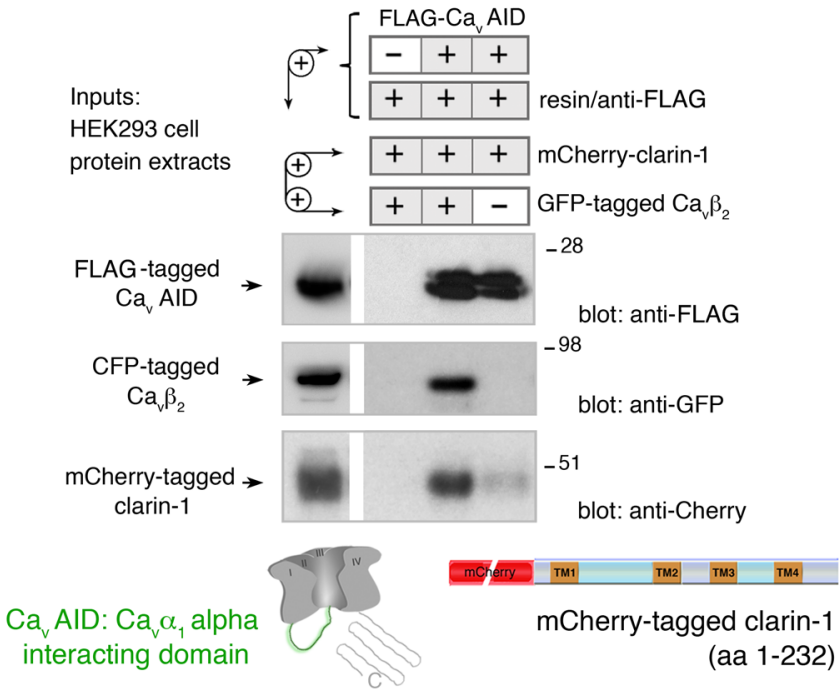

E

Clarin-1 and harmonin interaction

harmonin $b$
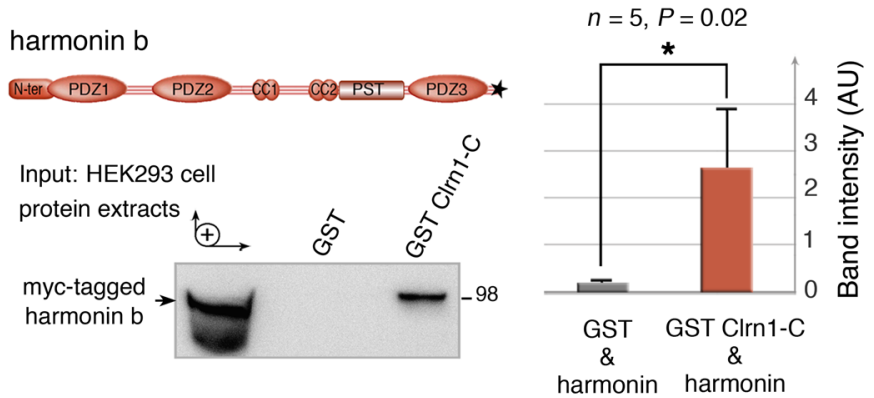

B

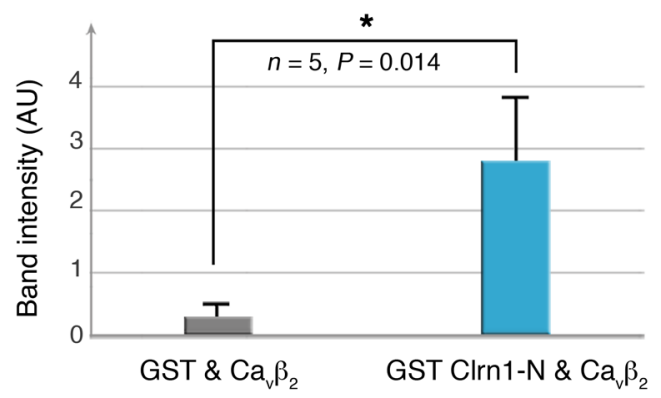

D

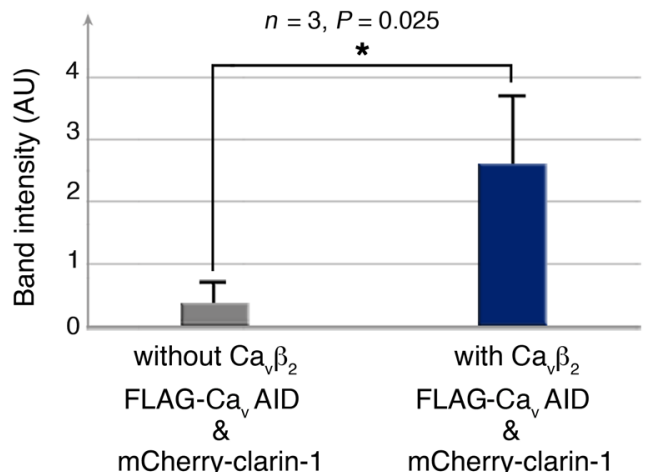

mCherry-clarin-1
F

Model of clarin-1, $\mathrm{Ca}_{v} \beta_{2}$, and harmonin interactions in the IHC presynaptic region

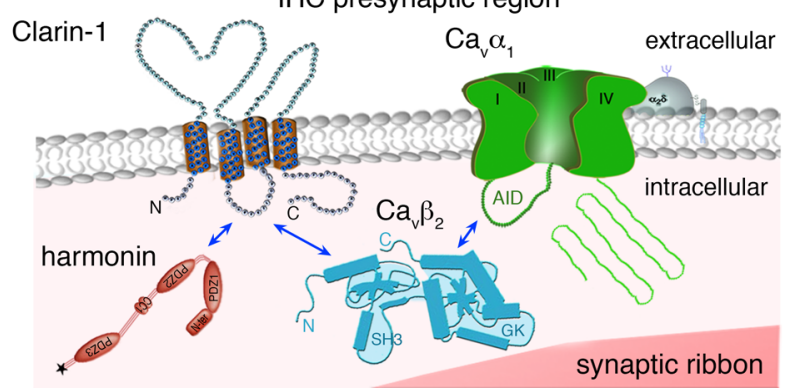

Figure 11. Interactions between clarin-1, the $\mathrm{Ca}_{\mathrm{v}} \mathbf{1 . 3}$ channel complex, and harmonin. (A and B) The GST-tagged clarin-1 N-terminal region (Clrn1-N) and C-terminal region (Clrn1-C) bind to CFP-tagged Ca $\beta_{2}$ produced in HEK293 cells, whereas CST alone does not. No binding is detected with the untagged Ca $\alpha_{1}$, CFP-tagged Rab11, or ECFP alone. (B) The bar chart indicates the significant interaction between clarin-1 and $\mathrm{Ca}_{\mathrm{v}} \beta_{2}\left(n=5,{ }^{*} P<0.05\right.$; unpaired Student's $t$ test). (C and D) In a coimmunoprecipitation assay, anti-FLAG M2 resin was incubated with HEK293 cells coproducing FLAG-tagged CavAID, mCherry-tagged clarin-1, and CFP-tagged $\mathrm{Ca}_{\mathrm{v}} \beta_{2}$, or mCherry-tagged clarin-1 and CFP-tagged $\mathrm{Ca}_{\mathrm{v}} \beta_{2^{*}}$ (D) Significant binding between $\mathrm{Ca}_{\mathrm{v}}$ AID and clarin- 1 is observed only in the presence of $\mathrm{Ca}_{\mathrm{v}} \beta_{2}\left(n=3,{ }^{*} P<0.05\right.$; unpaired Student's $t$ test). (E) CST-tagged Clrn1-C binds to myc-tagged harmonin b, whereas CST alone does not. The bar chart shows the significant interaction between clarin-1 and harmonin ( $n=5, P<0.05$; unpaired Student's $t$ test). (F) Schematic representation of a synaptic active zone summarizing the interactions between clarin-1, the $C a^{2+}$ channel subunits $C a_{v} \beta_{2}$ and $C a_{v} \alpha_{1}$, and harmonin (see uncut gels in supplemental material). 
Hearing rescue by virus-mediated gene transfer into the IHCs of clarin-1-deficient mice. Clrn1 $1^{-/}$mice lacking exon 1 have no apparent IHC synaptic defects (8), whereas $\mathrm{Ca}^{2+}$-mediated exocytosis was found here to be less efficient in the IHCs of Clrn $1^{\text {ex4-/- }}$ mice. We therefore investigated the possibility of these synaptic phenotypes being directly due to clarin-1 deficiency. We investigated whether the virus-mediated transfer of intact Clrn1 into the auditory hair cells could prevent synaptic defects and hearing decline in our 2 mouse models. We used a recombinant adeno-associated virus (AAV) carrying the mouse clarin-1 isoform $2 \mathrm{cDNA}$, encoding the predicted principal tetraspan glycoprotein of hair cells (Figure 1A), followed by an IRES-GFP sequence for the visualization of transduced cells (AAV2/8-Clrn1-IRES-GFP) (Figure 8A). This viral vector was microinjected, through the round window mem-

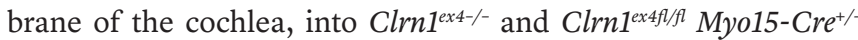
mice on P1-P3 (Figures 8-10). Viral transduction was detected in $90 \%$ of IHCs and $20 \%$ of OHCs on P14-P20 (Figure $8 \mathrm{~A}$ ). We first checked, in control experiments, that injections of AAV2/8-Clrn1IRES-GFP or AAV2/8-GFP into the inner ears of WT mice had no effect on hearing thresholds, and that the injection of AAV2/8-

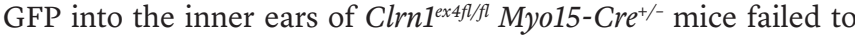
improve hearing sensitivity (Figure 8B).

In Clrn $1^{e x 4-1-}$ mice, hearing sensitivity in the AAV2/8-Clrn1IRES-GFP-injected ear was poor, with ABR thresholds at $10 \mathrm{kHz}$ and $20 \mathrm{kHz}$ averaging $83 \mathrm{~dB}$ and $98 \mathrm{~dB}$, respectively, whereas SPL values of $99 \mathrm{~dB}$ and $108 \mathrm{~dB}$ SPL, respectively, were obtained for the noninjected ears $(P<0.01$ for each comparison; Figure $8 C)$. Structural analyses 3 weeks after the injection showed that the hair bundles of IHCs and OHCs in the injected ears of Clrn $1^{\text {ex4-/- }}$ mice remained misshapen (Figure 8D). Interestingly, after similar injections of AAV2/8-Clrn1-IRES-GFP into the inner ears of Clrn1 $1^{\text {exffl/fl }} \mathrm{Myo15}-\mathrm{Cr}^{+/-}$mice, audiometric tests on P22-P24 showed an almost complete rescue of hearing, at all frequencies tested (Figure 9A): the ABR thresholds in the microinjected ears at $10 \mathrm{kHz}$ and $20 \mathrm{kHz}$ averaged $20 \mathrm{~dB}$ and $38 \mathrm{~dB}$, respectively (values similar to those obtained for control mice), as opposed to $67 \mathrm{~dB}$ and $80 \mathrm{~dB}$ for the noninjected ears $(P<0.001$ for each comparison). Significant hearing conservation was still observed at P60, with thresholds averaging $60 \mathrm{~dB}$ and $67 \mathrm{~dB}$ SPL at low and high frequencies, respectively, whereas Clrn1 ${ }^{\text {extfl/fl }} \mathrm{Myo15}_{\mathrm{C}} \mathrm{Cr}^{+/-}$mice that did not receive injections were profoundly deaf, with no measurable ABR waves at an SPL of $100 \mathrm{~dB}$ (Figure 9, B and C). Followup click ABR tests at $\mathrm{P} 60$ and $\mathrm{P} 120$ showed a progressive increase in hearing thresholds with aging in the AAV2/8-Clrn1-IRES-GFPtreated Clrn1 $1^{e x f f / f l}$ Myo15-Cre $e^{+/-}$mice (Supplemental Figure 3C), probably reflecting a slow progressive degradation of DPOAEs after P2O (Figure 1E), since viral transduction rates were low for OHCs ( 20\%; see Figure 8A). Nevertheless, at P120, the hair cells

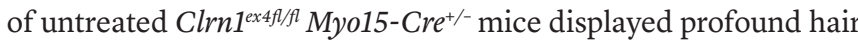
bundle disorganization, whereas stereociliary structures were well preserved in ears into which AAV2/8-Clrn1-IRES-GFP had been injected (Figure 9D and Supplemental Figure 3D).

Despite the poor protection of hearing function in Clrn $1^{\text {ext-1- }}$ mice injected with AAV2/8-Clrn1-IRES-GFP, due to the early development of stereociliary abnormalities, ribbon synapse organization and function were well preserved in these mice. Indeed, $\mathrm{Ca}^{2+}$ current characteristics, kinetics, the $\mathrm{Ca}^{2+}$ efficiency of synaptic exocytosis (Figure 10A), and the clustering of $\mathrm{Ca}_{\mathrm{v}} 1.3$ channels (Figure 10B) in the active zone of IHCs in P15-P18 Clrn1 $1^{\text {ext-/ }}$ mice injected with AAV2/8-Clrn1-IRES-GFP were identical to those of control IHCs (Supplemental Table 3). The ratio of the coimmunoreactive area (overlap between $\mathrm{Ca}_{\mathrm{v}} 1.3$ and ribeye staining) to the $\mathrm{Ca}_{\mathrm{v}} 1.3$-immunoreactive domain did not differ between control IHCs ( $64 \% \pm 3 \%, n=60$ ribbons) and the IHCs of Clrn $1^{\text {ex4- } /-}$ mice injected with AAV2/8-Clrn1-IRES-GFP $(57 \% \pm 3 \%, n=54$ ribbons; $P=0.22)$, whereas a significant difference was observed for the IHCs of untreated Clrn1 $1^{\text {ex4-/- }}$ mice (38\% $\pm 3 \%, n=89$ ribbons; $P<0.05)$. Thus, the re-expression of clarin-1 in the IHCs of clarin-1-deficient mice during the early postnatal period efficiently prevents the occurrence of synaptic defects caused by the absence of clarin- 1 .

Clarin-1 is a member of the L-type $\mathrm{Ca}^{2+}$ channel complex. Based on the mispositioning of the $\mathrm{Ca}_{\mathrm{v}} 1.3 \mathrm{Ca}^{2+}$ channels in the absence of clarin-1, we investigated the molecular basis of the links between clarin- 1 and components of the $\mathrm{Ca}_{\mathrm{v}} 1.3 \mathrm{Ca}^{2+}$ channel complex, such as the pore-forming subunit $\mathrm{Ca}_{\mathrm{v}} \alpha_{1}$ (aa 1-2,203, NP_058994.1) or the auxiliary subunit $\mathrm{Ca}_{\mathrm{v}} \beta_{2}$ (aa 2-604, NP_4466303.1). We tested a possible direct interaction between clarin- 1 and $\mathrm{Ca}_{\mathrm{v}} \alpha_{1}$ or $\mathrm{Ca}_{\mathrm{v}} \beta_{2}$ in pull-down experiments using 2 overlapping glutathione- $S$-transferase-tagged (GST-tagged) clarin-1 fusion proteins: Clrn1-N (aa 1-136) contains the N-terminal region encompassing the first 2 transmembrane domains (TM1 and TM2), and Clrn1-C (aa 121232) contains the $\mathrm{C}$-terminal region encompassing TM3 and TM4 (Figure 11A and Supplemental Figure 4A). Following incubation with appropriate protein extracts from HEK293 cells (Figure 11, A and B), a significant interaction was observed between GSTtagged Clrn1-N or Clrn1-C and cyan fluorescent protein-tagged (CFP-tagged) $\mathrm{Ca}_{\mathrm{v}} \beta_{2}(n=5, P<0.05)$. No interaction was observed between the 2 clarin- 1 fusion proteins and $\mathrm{Ca}_{\mathrm{v}} \alpha_{1}$, CFP-tagged Rab11, or CFP alone, the last 2 of these proteins being unrelated and used as negative controls (Figure 11, $\mathrm{A}$ and $\mathrm{B}$ ). $\mathrm{Ca}_{\mathrm{v}} \alpha_{1}$ has been shown to interact with $\mathrm{Ca}_{\mathrm{v}} \beta_{2}$ (26). We therefore investigated the possible formation of a tripartite complex between $\mathrm{Ca}_{\mathrm{v}} \alpha_{1}, \mathrm{Ca}_{\mathrm{v}} \beta_{2}$, and clarin-1. We used mCherry-tagged clarin-1, which was efficiently targeted to the plasma membrane in transfected HeLa cells (Supplemental Figure 4B). We incubated FLAG-tagged Ca_AID ( $\alpha$ interaction domain of $\mathrm{Ca}_{\mathrm{v}} \alpha_{1}$; aa 406-576, NP_058994.1), which is known to interact with $\mathrm{Ca}_{\mathrm{v}} \beta_{2}$, with protein extracts from transfected HEK293 cells producing mCherry-tagged clarin-1 alone or together with CFP-tagged $\mathrm{Ca}_{\mathrm{v}} \beta_{2}$ (Figure $11 \mathrm{C}$ ). An analysis of the proteins binding to the FLAG-tagged $\mathrm{Ca}_{\mathrm{V}} \mathrm{AID}$ immobilized on anti-FLAG magnetic beads showed that significant immunoprecipitation of mCherry-tagged clarin-1 occurred only in the presence of CFP-tagged $\mathrm{Ca}_{\mathrm{v}} \beta_{2}(n=3, P<0.05$; Figure 11, C and D). These findings indicate that clarin- 1 and $\mathrm{Ca}_{\mathrm{v}} \beta_{2}$ interact directly, facilitating the formation of a tripartite complex including $\mathrm{Ca}_{\mathrm{v}} \alpha_{1}$.

We then investigated the possible involvement of other IHC synaptic proteins in the protein complex containing $\mathrm{Ca}_{\mathrm{v}} 1.3$ channels and clarin-1. Clarin-1 contains a C-terminal class II PDZbinding motif (PBM type II). We therefore searched for a possible interaction of clarin-1 with the PDZ domain-containing protein harmonin, which is defective in Usher syndrome type IC and has also been shown to associate with $\mathrm{Ca}_{\mathrm{v}} 1.3$ channels $(27,28)$. Following incubation with protein extracts from transfected HEK293 
A EEBR waves in control and clarin-1-deficient mice

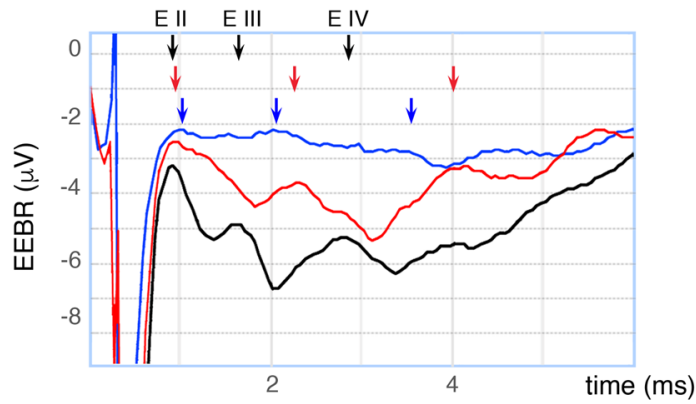

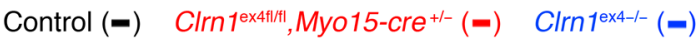

B Otoferlin and NF200 immunostaining of the cochlea of control and clarin-1-deficient P25 mice

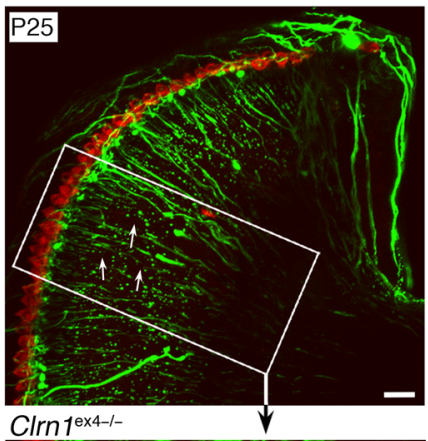

Apical region of a Clrn $1^{\text {ex4-/- }}$ cochlea
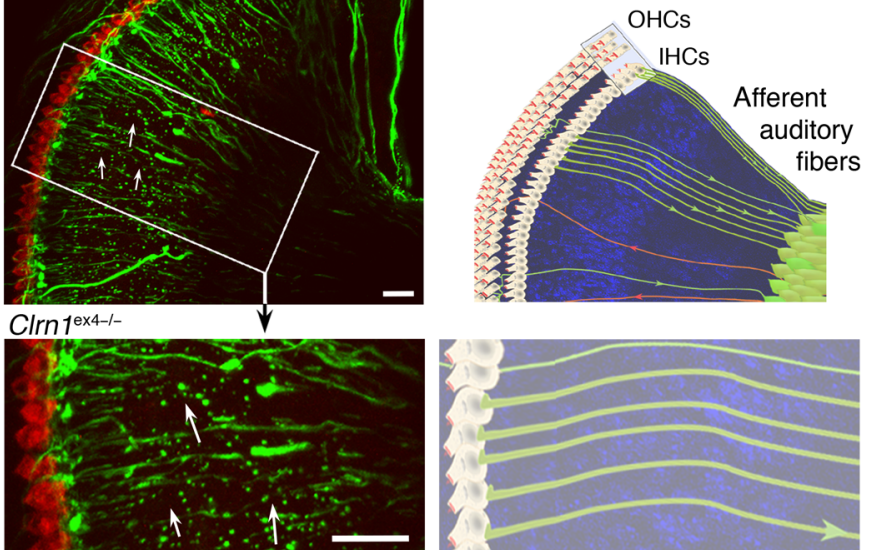

Control
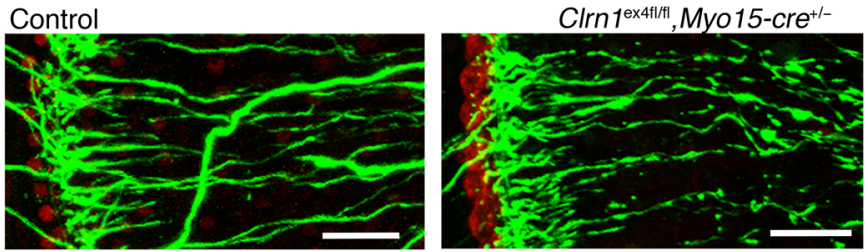

C Ribeye and GluA2 at the IHC synapse
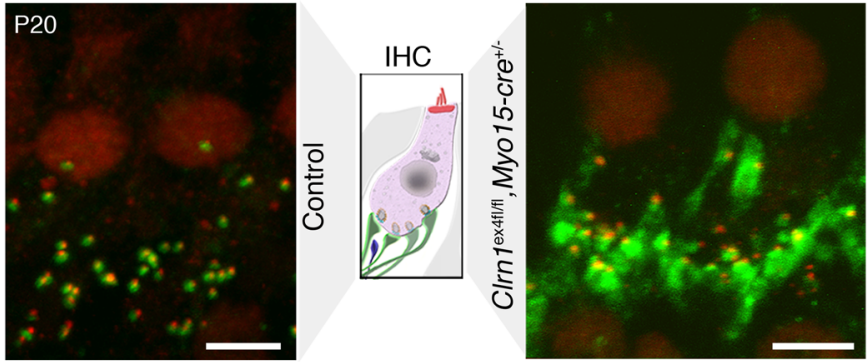

D $\quad$ HCs of control and clarin-1-deficient P28 mice
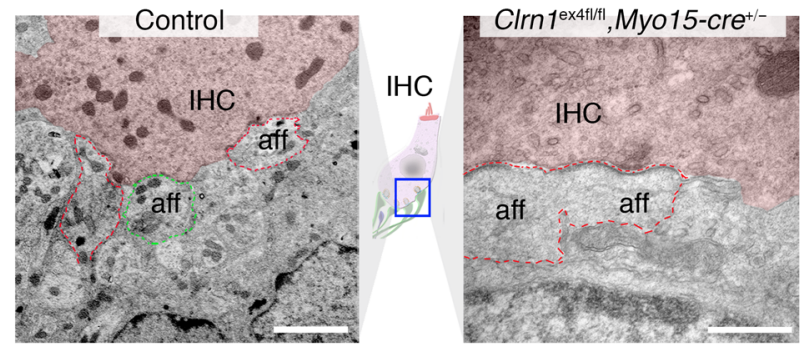

Disrupted postsynaptic regions in $\mathrm{Clrn} 1^{\mathrm{ex4-1-}}$ mice

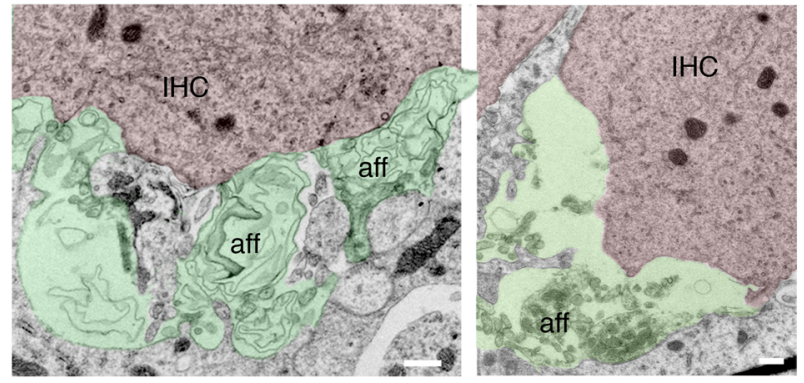

E The cochlear ganglion neurons in control and clarin-1deficient P25-P30 mice

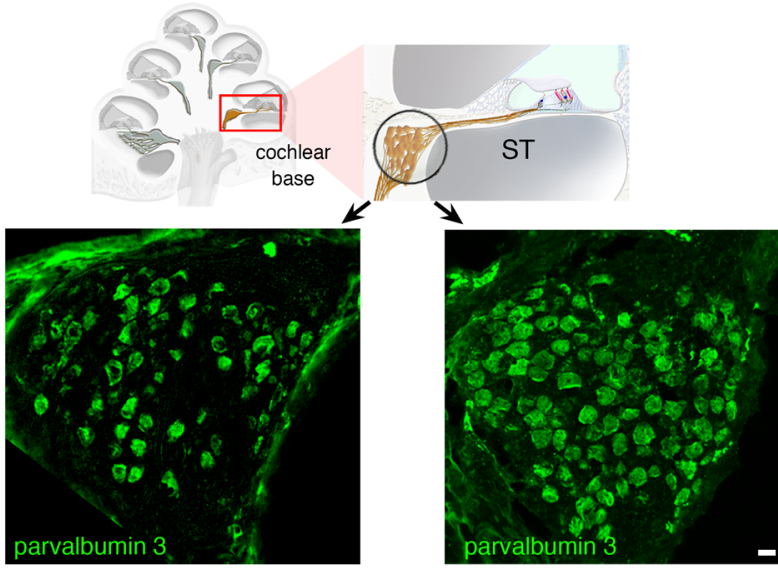

Clrn $1^{\text {ex4-l- }}$ mice

Control mice

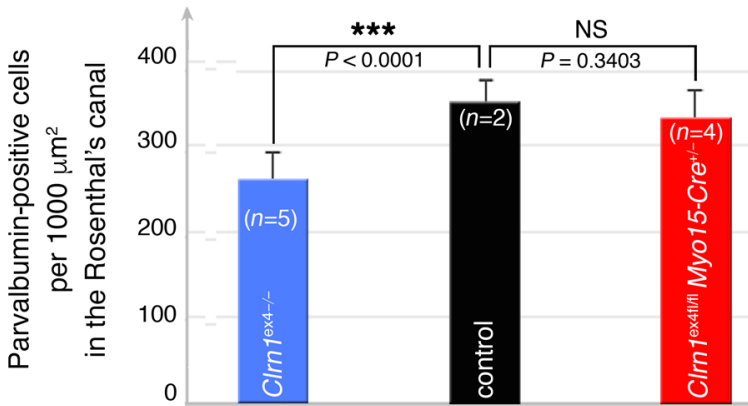

Figure 12. IHC postsynaptic defects in clarin-1-deficient mice. (A) Representative electrically evoked brainstem responses (EEBRs) in the cochleae of P2O control,

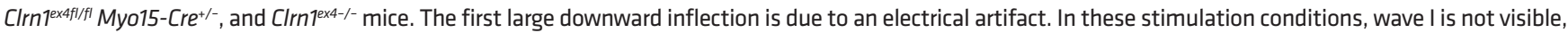
as signal processing by the IHC synapse is bypassed. EEBR wave II (EII) and later waves (EIII and EIV), corresponding to the responses of higher auditory centers, are clearly visible in the control mouse (black trace, representative of 10 mice), absent in Clrn ${ }^{\text {ex4-/- }}$ mice (blue trace, representative of 6 mice), and significantly delayed and reduced in Clrn $7^{\text {pxffl/fl }}$ Myo15-Cre ${ }^{+/-}$mice (red trace, representative of 10 mice). (B) Cochleae immunostained with Neurofilament 200 (NF200; green) showing a significant loss of auditory nerve fibers in P25 Clrn 1ex4-/- mice (arrows) relative to control and Clrn 7ex4f//fl Myo15-Cre ${ }^{+/-}$mice of the same age. (C) IHCs of P2O

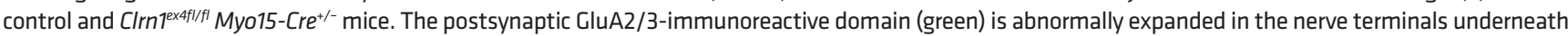
the IHCs of Clrn7 ${ }^{\text {pxffl/fl }}$ Myo15-Cre ${ }^{+/-}$mice $(n=7)$. (D) Representative micrographs of the IHC synaptic region highlighting the expansion of postsynaptic terminals in Clrn pexff/fl/ Myo15-Cre ${ }^{+/-}$mice (red dotted line; representative of 3 mice and $10 \mathrm{IHCs}$ ) and the swelling (artificially colored in green) of the postsynaptic boutons in Clrn 12x4-/ mice (representative of 3 mice and $10 \mathrm{IHCS}$ ) as compared with control mice (representative of 3 mice and $13 \mathrm{IHCS}$ ). (E) Quantification of the loss of parvalbumin-positive neurons in Rosenthal's canal in control (4 cochleae from 3 mice), Clrn $7^{\text {px4-/- }}$ (8 cochleae from 4 mice), and Clrn $7^{\text {exffl/fI }}$ Myo15-Cre ${ }^{+/-}$mice (6 cochleae from 3 mice) (values are mean \pm SD; ANOVA, post hoc Holm-Sidak test). Scale bars: $500 \mathrm{~nm}$ (D), $20 \mu \mathrm{m}$ (B and E), $5 \mu \mathrm{m}$ (C). 
A Clarin-1 in the IHC presynaptic and postsynaptic regions
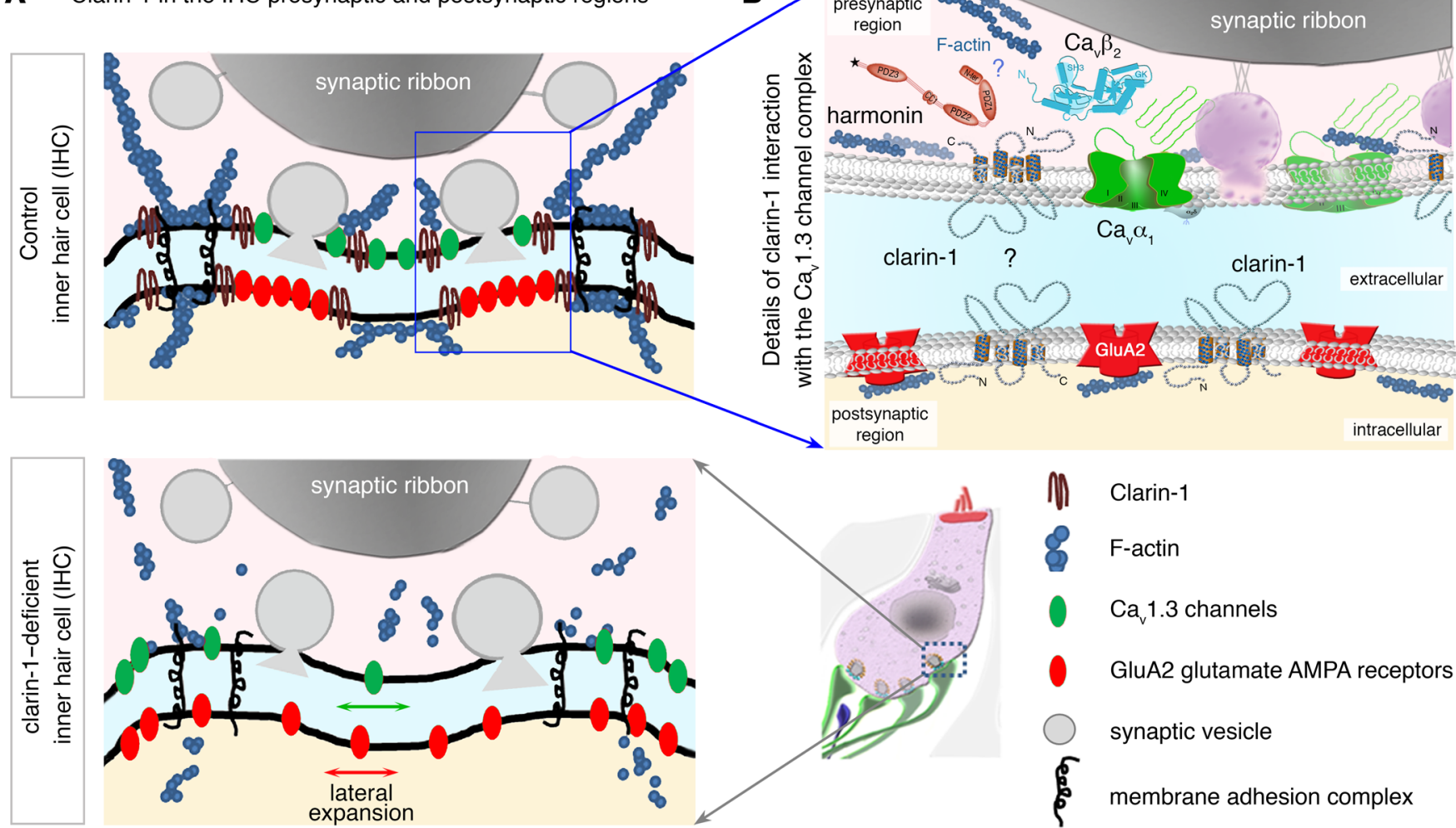

Clarin-1

F-actin

Ca 1.3 channels

GluA2 glutamate AMPA receptors

synaptic vesicle

membrane adhesion complex

Figure 13. Clarin-1 is required for the compact organization of presynaptic $\mathrm{Ca}_{\mathrm{v}} \mathbf{1 . 3} \mathrm{Ca}^{2+}$ channels and postsynaptic GluA2/3 AMPA receptors. (A) Schematic diagram of an IHC synaptic active zone in control (top panel) and clarin-1-deficient (bottom panel) mice. In the presynaptic active zone of release, clarin-1 interacts with $\mathrm{Ca}_{\mathrm{v}} \beta_{2}$, harmonin, and the F-actin cytoskeleton to cluster $\mathrm{Ca}_{\mathrm{v}} 1.3 \mathrm{Ca}^{2+}$ channels close to the synaptic vesicles. The lack of clarin-1 results in a dislocation of the synaptic F-actin network associated with a spatial disorganization of both presynaptic Ca 1.3 channels and postsynaptic GluA2/3 glutamate receptors. (B) Schematic representation of molecular interactions between clarin-1 and other proteins in the IHC synaptic active zone. Clarin-1 may be required for the trans-synaptic alignment of the $C a_{v} 1.3$ channel complex (harmonin-Cav $\beta_{2}-C a_{v} \alpha_{1}$ ) and postsynaptic glutamate receptors.

cells producing the myc-tagged harmonin protein (aa 2-852, AAM44072.1), GST-tagged Clrn1-C bound to harmonin, whereas GST alone did not $(n=5, P<0.05$; Figure $11 \mathrm{E})$.

Thus, clarin-1 binds to the submembrane auxiliary subunit $\mathrm{Ca}_{\mathrm{v}} \beta_{2}$ and the scaffold protein harmonin, 2 proteins of the $\mathrm{Ca}_{\mathrm{v}} 1.3$ channel complex (Figure 11F).

The loss of clarin-1 in IHCs results in postsynaptic defects. Clrn1 is also expressed by the primary auditory neurons synapsing with IHCs $(1,7)$. We investigated the possible effect of an absence of clarin-1 on postsynaptic auditory nerve fibers, by recording electrically evoked brainstem responses (EEBRs) following direct stimulation of the primary auditory neurons. No significant EEBR waves were evoked in P2O Clrn $1^{\text {ext-/- }}$ mice $(n=6)$, indicating a strong electrical conduction defect in primary auditory neurons lacking clarin-1 (Figure 12A). On P2O, in Clrn $1^{\text {exffl/fl }} \mathrm{Myo15}-\mathrm{Cre}^{+/-}$mice, which lack clarin-1 only in the presynaptic hair cells, EEBR waves were, surprisingly, of smaller amplitude than those of control mice, and the peaks of waves II (EII) and III (EIII) were significantly delayed ( $n=10$; Figure 12A). Clarin-1 expression in IHCs is, thus, also important for the maintenance of normal electrical conduction in primary auditory nerve fibers.

Immunofluorescence and ultrastructural analyses revealed the existence of various postsynaptic abnormalities in the absence of clarin-1. These synaptic abnormalities differed in

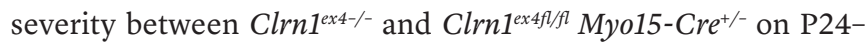
P30. Immunostaining for neurofilaments revealed a regression of auditory neuronal fibers along the spiral cochlea in Clrn1 $1^{\text {ext-/- }}$ mice (Figure 12B). The distribution of postsynaptic GluA2/3 AMPA receptors at the distal end of the dendrites of primary auditory neurons was altered in the 2 types of clarin-1-deficient mice. In Clrn1 ${ }^{\text {ex } 4 f / f l}$ Myo15-Cre ${ }^{+/-}$mice on P24-P30, abnormally enlarged GluA2/3-immunoreactive territories were observed (Figure 12C and Supplemental Figure 5). In control IHC ribbon synapses, the postsynaptic AMPA receptors normally formed small patches, less than $1 \mu \mathrm{m}$ across, facing each ribbon and its cluster of $\mathrm{Ca}^{2+}$ channels across the synapse $(29,30)$. Transmission electron microscopy confirmed the expansion of the postsynaptic region and revealed an occasional loss of dendrite ends (Figure 12, B and D). Ultrastructural analyses of P28 Clrn1 $1^{\text {ex4-/- }}$ mice revealed an abnormal swelling of IHC afferent terminals ( $n=3$ mice and 10 IHCs) and enlarged postsynaptic afferent terminals in Clrn1 ${ }^{\text {exffl/fl }} \mathrm{Myo15}-\mathrm{Cre}^{+/-}$(representative of 3 mice and 10 IHCs) relative to control mice ( $n=3$ mice and 13 IHCs; Figure 12D). The observed morphological defects resembled the postsynaptic afferent swelling observed during noise trauma or intracochlear perfusion with AMPA receptor agonists (31). We investigated the loss of auditory neurons in the absence of clarin-1 further, by counting the parvalbumin-positive neurons 
in the Rosenthal's canals of control, Clrn1 $1^{\text {ext-/-}}$, and Clrn1 $1^{\text {ex4f/ft }}$ Myo15-Cre ${ }^{+/-}$mice on P24-P30 (Figure 12E). Neuron density in the mid-base region decreased from $346.8 \pm 16.7$ neurons per $1,000 \mu \mathrm{m}^{2}$ ( $n=4$ cochleae from 3 mice $)$ in controls to $259.6 \pm 8.9$ neurons per $1,000 \mu^{2}(n=8$ cochleae from 4 mice $)$ in Clrn $1^{\text {ext- }-}$ mice $(P<0.001$, mean \pm SD; ANOVA, post hoc Holm-Sidak test; Figure 12E). No significant neuron loss was detected in Clrn $1^{\text {ex } 4 \mathrm{f} / \mathrm{fl}}$ Myo15-Cre ${ }^{+/-}$mice, which had $329.5 \pm 10.7$ neurons per 1,000 $\mu \mathrm{m}^{2}$ ( $n=6$ cochleae from 3 mice; $P>0.05$; Figure $12 \mathrm{E}$ ).

Our results show that the postnatal hair cell-specific elimination of clarin-1, which causes a disorganization of the presynaptic $\mathrm{Ca}_{\mathrm{v}} 1.3$ channel complex in the active zone of IHCs, also results in postsynaptic afferent nerve defects: (a) morphological changes in the afferent boutons, associated with a disorganization of the AMPA receptors; and (b) defective electrical conduction in the primary auditory neurons, probably partly due to a loss of afferent nerve fibers.

\section{Discussion}

Clarin-1, a tetraspan protein defective in Usher syndrome type IIIA (deaf-blindness), has been reported to be involved in hair bundle morphogenesis $(7,8,32)$. We show here that a deletion of clarin-1 leads to a lower $\mathrm{Ca}^{2+}$ efficiency of exocytosis in IHCs, probably due to abnormal clustering of $\mathrm{Ca}^{2+}$ channels in the active zones of the ribbon. We suggest that the binding of clarin- 1 to the $\mathrm{Ca}_{\mathrm{v}} \beta_{2}$ auxiliary subunit and the PDZ domain-containing protein harmonin couples the $\mathrm{Ca}_{\mathrm{v}} 1.3 \mathrm{Ca}^{2+}$ channel complex to the IHC exocytotic machinery. Virus-mediated Clrn1 transfer to mutant hair cells reversed the synaptic defects and restored hearing. These results reveal a new key role for clarin-1 at the IHC ribbon synapse, but they also raise important questions about the rehabilitation of hearing in USH3A patients.

Clarin-1 as a key component of the hair bundle and ribbon synapse of auditory hair cells. The presence of hair bundle abnormalities at birth in Clrn1 $1^{\text {ext-/- }}$ mice, and from P3O onward in Clrn1 ${ }^{\text {exufl/fl }} \mathrm{Myo15}-\mathrm{Cre}^{+/-}$mice, clearly indicates that clarin-1 is essential for both the development and maintenance of normal auditory hair bundle structure and function. These findings are consistent with previous reports on $\mathrm{Clrn1}^{-/-}$total knockout mice obtained by deletion of Clrn1 exon $1(7,8)$. Our detailed analysis of both Clrn $1^{\text {ex4-/- }}$ and Clrn $1^{\text {exfl/fl }} \mathrm{Myo15-Cre^{+/- }}$ mice also highlighted an additional essential role of clarin-1 at the auditory IHC ribbon synapses in the spatial organization and regulation of $\mathrm{Ca}_{\mathrm{v}} 1.3 \mathrm{Ca}^{2+}$ channels (Figure 13). Synaptic abnormalities appeared only after $\mathrm{P} 9$, close to the time of hearing onset at P12, in both Clrn $1^{\text {ex4-/- }}$ and Clrn $1^{\text {ex4fl/fl }} \mathrm{Myo15}^{-\mathrm{Cr}^{+/-}} \mathrm{IHCs}$, indicating a requirement of clarin-1 for IHC synapse maturation and/ or maintenance, but not for IHC synapse formation. A recent study of $\mathrm{Clrn1}^{-/-}$mutants in the A/J mouse genetic background showed that these mice had impaired vision, probably due to the unique function of clarin-1 in synaptic transmission between the light-sensitive photoreceptor cells and their associated neurons (33). Electroretinogram measurements in these mice revealed a normal A wave response (indicative of a normal phototransduction machinery), with a significantly smaller B wave than observed in WT mice, consistent with lower levels of synaptic transmission between rod photoreceptor cells and their associ- ated neurons (33). The virus-mediated postnatal delivery of a clarin-1 transcript to IHCs in the inner ear in vivo prevented the occurrence of synaptic defects in Clrn $1^{\text {ext-/- }}$ mice, demonstrating a direct causal link between clarin-1 deletion and IHC synaptic failure. By contrast, clarin-1 supplementation in Clrn $1^{\text {ext-/- }}$ mice did not correct the early developmental disorganization of the hair bundles, indicating that the synaptic function of clarin-1 is independent of its role in the hair bundle.

Clarin-1 is required for the tight clustering of presynaptic $C a_{V} 1.3$ channels. Our physiological, morphological, and molecular data indicate that, at time points close to hearing onset in mice, clarin- 1 is critical for the establishment of tightly clustered $\mathrm{Ca}_{\mathrm{v}} 1.3$ channels at the synaptic ribbon. We found that the absence of clarin-1 resulted in a marked disruption of the F-actin network at the IHC ribbon synapse similar to that observed at the apical surface of the cuticular plate and the stereocilia. Could the disruption of $\mathrm{F}$-actin be responsible for the spatial disorganization of the $\mathrm{Ca}_{\mathrm{v}} 1.3 \mathrm{Ca}^{2+}$ channels? It has been suggested that a direct association between $\mathrm{Ca}_{\mathrm{v}} \beta_{2}$ and $\mathrm{F}$-actin promotes the directional transport of $\mathrm{Ca}^{2+}$ channels to the plasma membrane of cardiomyocytes (34), and, at the ribbon synapse of salamander photoreceptors, the confinement of $\mathrm{Ca}^{2+}$ channels beneath the ribbon is regulated by actin and membrane cholesterol (35). The disruption of F-actin with latrunculin-A in WT IHCs has been shown to disrupt the spatial distribution of $\mathrm{Ca}_{\mathrm{v}} 1.3 \mathrm{Ca}^{2+}$ channels (23) in a manner similar to that observed here, in the active zones of IHCs lacking clarin- 1 from the 2 types of mutant mice.

Our biochemical analyses showed that clarin-1 could physically bind harmonin and $\mathrm{Ca}_{\mathrm{v}} \beta_{2}$, this channel subunit subsequently facilitating the formation of a tripartite complex including the pore-forming subunit of $\mathrm{Ca}_{\mathrm{v}} 1.3 \mathrm{Ca}^{2+}$ channels, $\mathrm{Ca}_{\mathrm{v}} \alpha_{1}$. Consistent with these results, IHCs lacking harmonin in $d f c r$ mice also have greater $\mathrm{Ca}^{2+}$ currents, with a negative shift in voltage-dependence, and a clustering defect of $\mathrm{Ca}_{\mathrm{v}} 1.3 \mathrm{Ca}^{2+}$ channels $(27,28)$. Based on the observed interactions of clarin-1 (Figure 11E), we suggest that clarin-1, harmonin, and $\mathrm{Ca}_{\mathrm{v}} 1.3$ channels, via their $\mathrm{Ca}^{2+}$ channel subunit $\mathrm{Ca}_{\mathrm{v}} \beta_{2}$, form a molecular platform associated with $\mathrm{F}$-actin in the active zones of the IHC. By confining the $\mathrm{Ca}_{\mathrm{v}} 1.3$ channels in the IHC active zone below the ribbon, this platform would produce overlapping $\mathrm{Ca}^{2+}$ domains and synchronize the fusion of multiple synaptic vesicles in the active zone (Figure 13).

An absence of clarin-1 also results in postsynaptic defects. Mirroring the expansion of the $\mathrm{Ca}_{\mathrm{v}} 1.3$ channels in the IHC active zone, an abnormal distribution of postsynaptic AMPA glutamate receptors was also observed in the absence of clarin-1, along with structural changes, such as the fusion and/or swelling of afferent dendrites. Could the postsynaptic defects observed in Clrn $1^{e x 4 f / f l}$ Myo15-Cre ${ }^{+/-}$mice result purely from disorganization of the presynaptic $\mathrm{Ca}_{\mathrm{v}} 1.3$ complex? This seems unlikely, because an absence of the presynaptic cytomatrix protein bassoon (36), $\mathrm{Ca}_{\mathrm{v}} \beta_{2}$ $(37)$, or harmonin $(27,28)$ in IHCs disorganizes the $\mathrm{Ca}_{\mathrm{v}} 1.3$ channels without affecting the postsynaptic clustering of glutamate receptors. As an alternative hypothesis, clarin-1 may be a member of pre- and postsynaptic membrane focal adhesion complexes at the IHC ribbon synapse, possibly interacting with proteins known to be involved in the extracellular matrix at neuronal synapses, such as thrombospondins (38), or in membrane-membrane synaptic adhesion, such as Eph/ephrins, cadherins, and the neurex- 
in/neuroligin proteins (39). Defects of the presynaptic scaffold protein Syd1 (synaptic-defective-1) at the neuromuscular junction result in abnormal presynaptic neurexin targeting, impairing the postsynaptic density cluster organization of neuroligin- 1 and glutamatergic receptors (40). A role for the auxiliary $\mathrm{Ca}^{2+}$ channel subunit $\mathrm{Ca}_{\mathrm{v}} \alpha_{2} \delta_{2}$ in the trans-synaptic coupling of IHC Ca 1.3 channels with the postsynaptic AMPA receptor complex has also been recently proposed (41). Based on our findings in clarin-1deficient mice, we suggest that clarin- 1 is a member of the transsynaptic membrane interaction adhesion complex at the IHC ribbon synapse, a complex essential for the focal alignment of the presynaptic $\mathrm{Ca}_{\mathrm{v}} 1.3$ channels and release sites with the postsynaptic AMPA receptors (Figure 13B).

Impact on the clinical exploration and treatment of USH3. In humans, despite a number of disabling CLRN1 mutations identified in USH3A patients, audiometric tests, essentially ABR measurements, indicate that the hair cells differentiate and function normally for long periods of time $(42,43)$. Indeed, almost all USH3A patients develop normal speech, and, although an elevation of hearing thresholds is diagnosed in most patients before the age of 10 years, some patients display only mild to moderate hearing threshold elevation at the time of detection, at an age of 30-40 years $(42,43)$. The discrepancy in hair cell phenotype between humans $(42,43)$ and Clrn1 total knockout mice $(7,11)$ suggests that compensatory mechanisms, possibly involving genes encoding other members of the clarin family (CLRN2 and CLRN3), operate much more efficiently, particularly during embryogenesis and early postnatal development, in humans than in mice. USH1 and USH 2 patients present congenital hearing loss. By contrast, there is a therapeutic window in USH3 patients during which potential therapeutic strategies for preventing or halting hearing decline can be tested. In Clrn $1^{e x 4 f / f l} \mathrm{Myo15}-\mathrm{Cr} \mathrm{C}^{+/-}$mice, hearing impairment was also progressive, starting as early as P15 and reaching profound deafness at P60. These features closely match the progressiveness of hearing loss in USH3A patients. Progressive hearing loss has also been reported for $\mathrm{Clrn}^{-/-}$total knockout mice in which clarin-1 was transiently expressed in the hair cells, under the control of the Atoh1 promoter, between E12.5 and P3; these mice were referred to as $\mathrm{KO}-\mathrm{TgAC} 1$ mice (32). Interestingly, a single cochlear injection of AAV2/8-Clrn1 (isoform 2) into KO-TgAC1 mice or our conditional Clrn1 $1^{e x 4 f / f l} \mathrm{Myo15}-\mathrm{Cr}^{+/-}$mice durably preserved the hair cells and hearing.

Cochlear implants are currently the only medical option available for treating severe to profound deafness in USH3A patients. These prosthetic devices consist of electrode arrays that bypass the hair cells and stimulate the primary auditory neurons directly. However, very little is known about the long-term performance of cochlear implants, as the number of spiral ganglion neurons progressively declines with aging in these patients. The significant decrease in the number of spiral ganglion neurons in Clrn $1^{\text {ext- }}$ mice and the delayed EEBR waves observed here in Clrn1 ${ }^{\text {exffl/fl }} \mathrm{Myo15-Cre}{ }^{+/-}$mice, if replicated in USH3A patients, would result in a poor long-term efficacy of cochlear implants. Considerable variability and poor performances have indeed been reported for cochlear implants in USH3A patients (ref. 44 and Claes Moller, Örebro University, personal communication), but there are currently no detailed and long-term follow-up analyses of pre- and postimplant performance scores for USH3A patients. Our study, showing the effective preservation of ribbon synapses and hair bundle structures following virus-mediated Clrn1 transfer into Clrn ${ }^{\text {exffl/fl }} \mathrm{Myo15}-\mathrm{Cr}^{+/-}$mice, indicates that a gene therapy approach targeting the hair cells would improve hearing. Our results, like those recently obtained for Usher syndrome type IC (45) and type IG (46), confirm that gene therapy approaches should treat genetic inner ear disorders very effectively. Future studies targeting both hair cells and neurons in clarin-1-deficient mice are, however, required, to obtain insight into how best to preserve cochlear neurons over time in the absence of clarin-1, which would be of great benefit in USH3A patients fitted with cochlear implants.

\section{Methods}

Generation of clarin-1-deficient mice. To engineer clarin-1-deficient mice, we used the Cre-lox system to delete Clrn1 exon 4 (Figure 1, B and C). The Clrn $1^{e x 4 f / f l}$ mice carrying the loxP sites on both alleles were crossed with $\mathrm{PGK}-\mathrm{Cre}^{+/}$mice to obtain a precocious and ubiquitous suppression of clarin-1 (Clrn1 $1^{\text {ext- }}$ mice; official nomenclature from Mouse Genome Informatics [MGI]: symbol, Clrn1 ${ }^{\text {tm1.2ugpa }}$; accession ID, 6099052), and with Myo15-Cre $e^{+-}$mice $(16,17)$ to specifically target clarin-1 suppression to hair cells at postnatal stages (Clrn1 ${ }^{\text {ex4f/fl/l }}$ Myo15$\mathrm{Cre}^{+/-}$mice; Clrn1 ${ }^{\text {tm1.lugpa }}$, MGI accession ID 6099051).

RT-PCR for isoform specificity. For RT-PCR analyses, freshly dissected inner ears were collected from P15 or P30 WT and Clrn1 $1^{\text {extff/fl }}$ Myo15-Cre ${ }^{+/-}$mice. Organs of Corti and inner hair cells (IHCs) were microdissected separately and quickly frozen in liquid nitrogen and stored at $-80^{\circ} \mathrm{C}$ until processing. Total RNA (100 ng) was reverse-transcribed with the SuperScript III One-Step RT-PCR system (Invitrogen) as described by the manufacturer using various isoform-specific primers (see Supplemental Methods).

For expression vectors, antibodies, protein-protein interactions (47), immunofluorescence (48), and electron microscopy analyses (49), see Supplemental Methods.

In vivo auditory tests. Auditory brainstem responses (ABRs), compound action potentials (CAPs), and distortion-product otoacoustic emissions (DPOAEs) were recorded in anesthetized mice, and the results were analyzed as previously described (50) (also see Supplemental Methods).

Hair cell electrophysiology. All IHC recordings were performed in the $20 \%-40 \%$ normalized distance from the apex, an area coding for frequencies ranging from 8 to $16 \mathrm{kHz}$. For IHC electrophysiological recordings, mouse organs of Corti, at different stages of postnatal development, were bathed at room temperature $\left(22^{\circ} \mathrm{C}-24^{\circ} \mathrm{C}\right)$ in an extracellular solution, with the following composition: $135 \mathrm{mM} \mathrm{NaCl}$, $5.8 \mathrm{mM} \mathrm{KCl}, 5 \mathrm{mM} \mathrm{CaCl}_{2}, 0.9 \mathrm{mM} \mathrm{MgCl}_{2}, 0.7 \mathrm{mM} \mathrm{NaH}_{2} \mathrm{PO}_{4}, 5.6 \mathrm{mM}$ glucose, $2 \mathrm{mM}$ Na-pyruvate, $10 \mathrm{mM}$ HEPES, $250 \mathrm{nM}$ apamin, 0.5 $\mu \mathrm{M}$ XE-991, $\mathrm{pH}$ 7.4. $\mathrm{Ca}^{2+}$ currents and membrane capacitance were recorded in the whole-cell voltage-clamp configuration, using 3- to 5-M $\Omega$-resistance pipettes with an EPC 10 amplifier and Patchmaster software (HEKA Elektronik) as previously described (19, 23).

Gene transfer through the cochlear round window membrane. A recombinant adeno-associated virus (AAV) carrying the mouse Clrn1 followed by an IRES-GFP sequence (AAV2/8-Clrn1-IRES-GFP) was microinjected through the round window membrane on anesthetized P1-P3 mice. The virus was packaged and titrated by Penn Medi- 
cine Vector Core (University of Pennsylvania School of Medicine, Philadelphia Pennsylvania, USA). Microinjections were performed on the left ear as previously described (46).

For quantification of in vivo injections of AAV2/8-Clrn1-IRESGFP, we captured high-magnification images along the cochlea at exactly the same distance from the base for all cochleae. Then, in each case, we manually counted all visible inner and outer hair cells and determined the percentage of GFP-positive cells.

Statistics. For in vivo recordings (data expressed as mean \pm SD or SEM as indicated), statistical significance of the differences observed was tested with Student's $t$ test, 2-way ANOVA coupled to the Bonferroni post hoc test (2-way ANOVA), or 2-tailed unpaired $t$ test with Welch's correction using Prism software (GraphPad). For ex vivo recordings, the data (expressed as mean \pm SEM) were analyzed with Origin (Microcal) and Igor software (WaveMetrics). Statistical significance is indicated in the figures; NS, not significant $(P>0.05)$; ${ }^{*} P<0.05,{ }^{* *} P<0.01,{ }^{* *} P<0.001$.

Study approval. All the experiments on mice were carried out according to protocols approved by the Animal Use Committees of INSERM, the University of Bordeaux (CEEA50), and Institut Pasteur (CETEA-2014-0032).

\section{Author contributions}

$\mathrm{DD}, \mathrm{CP}$, and AEA designed research studies and wrote the manuscript. DD, AEA, SP, PP, MC, PFYV, MT, AE, AT, YB, VM, SD, AA, EP, OAP, OA, LL, SS, and PA conducted experiments and analyzed data.

\section{Acknowledgments}

We thank Jean-Pierre Hardelin and Jacques Boutet de Monvel for critical reading of the manuscript. SP and MC benefited from fellowships from the Ministry of National Education, Research and Technology of France, and PP from the European Union's Horizon 2020 Marie Sklodowska-Curie grant 665807. This work was supported by European Research Council (ERC) advanced grant "Hair bundle" (ERC-2011-AdG 294570), the European Union Seventh Framework Programme under grant agreement HEALTH-F2-2010-242013 (TREATRUSH), the French National Research Agency (ANR) as part of the second "Investissements d'Avenir" programme (light4deaf, ANR-15-RHUS-0001), ANRHearInNoise-(ANR-17-CE16-0017), LHW-Stiftung, FAUN Stiftung (Suchert Foundation), LABEX Life-senses (ANR-10LABX-65), and a grant from the Fondation Pour l'Audition to DD (2015-APA Research Grant).

Address correspondence to: Didier Dulon, INSERM UMRS1120, Université de Bordeaux, Laboratoire de Neurophysiologie de la Synapse Auditive, Bordeaux Neurocampus, 33076 Bordeaux, France. Phone: 33.9.61.49.81.50; Email: didier.dulon@inserm. fr. Or to: Christine Petit, Unité de Génétique et Physiologie de l'Audition, Institut Pasteur, 25 rue Dr Roux, 75015 Paris, France. Phone: 33.1.45.68.88.90; Email: christine.petit@pasteur.fr. Or to: Aziz El-Amraoui, Unité de Génétique et Physiologie de l'Audition, Institut Pasteur, 25 rue Dr Roux, 75015 Paris, France. Phone: 33.1.45.68.88.92; Email: aziz.el-amraoui@pasteur.fr.
1. Adato A, et al. USH3A transcripts encode clarin-1, a four-transmembrane-domain protein with a possible role in sensory synapses. Eur J Hum Genet. 2002;10(6):339-350.

2. Fields RR, et al. Usher syndrome type III: revised genomic structure of the USH3 gene and identification of novel mutations. Am J Hum Genet. 2002;71(3):607-617.

3. Västinsalo $\mathrm{H}$, et al. Alternative splice variants of the USH3A gene Clarin 1 (CLRN1). Eur J Hum Genet. 2011;19(1):30-35.

4. Joensuu $\mathrm{T}$, et al. Mutations in a novel gene with transmembrane domains underlie Usher syndrome type 3. Am J Hum Genet. 2001;69(4):673-684.

5. Isosomppi J, Västinsalo H, Geller SF, Heon E, Flannery JG, Sankila EM. Disease-causing mutations in the CLRN1 gene alter normal CLRN1 protein trafficking to the plasma membrane. $\mathrm{Mol}$ Vis. 2009;15:1806-1818.

6. Zallocchi M, et al. Localization and expression of clarin-1, the Clrn1 gene product, in auditory hair cells and photoreceptors. Hear Res. 2009;255(1-2):109-120.

7. Geng R, et al. Usher syndrome IIIA gene clarin-1 is essential for hair cell function and associated neural activation. Hum Mol Genet. 2009;18(15):2748-2760.

8. Geng R, et al. The mechanosensory structure of the hair cell requires clarin-1, a protein encoded by Usher syndrome III causative gene. J Neurosci. 2012;32(28):9485-9498.

9. Ogun O, Zallocchi M. Clarin-1 acts as a modulator of mechanotransduction activity and presynaptic ribbon assembly. JCell Biol. 2014;207(3):375-391.
10. Gopal SR, et al. Zebrafish models for the mechanosensory hair cell dysfunction in Usher syndrome 3 reveal that clarin- 1 is an essential hair bundle protein. J Neurosci. 2015;35(28):10188-10201.

11. Geller SF, et al. CLRN1 is nonessential in the mouse retina but is required for cochlear hair cell development. PLoS Genet. 2009;5(8):e1000607.

12. Phillips JB, Västinsalo H, Wegner J, Clément A, Sankila EM, Westerfield M. The cone-dominant retina and the inner ear of zebrafish express the ortholog of CLRN1, the causative gene of human Usher syndrome type 3A. Gene Expr Patterns. 2013;13(8):473-481.

13. Constals A, et al. Glutamate-induced AMPA receptor desensitization increases their mobility and modulates short-term plasticity through unbinding from Stargazin. Neuron. 2015;85(4):787-803.

14. Hafner AS, et al. Lengthening of the stargazin cytoplasmic tail increases synaptic transmission by promoting interaction to deeper domains of PSD-95. Neuron. 2015;86(2):475-489.

15. Lallemand Y, Luria V, Haffner-Krausz R, Lonai P. Maternally expressed PGK-Cre transgene as a tool for early and uniform activation of the Cre site-specific recombinase. Transgenic Res. 1998;7(2):105-112.

16. Caberlotto E, et al. Usher type $1 G$ protein sans is a critical component of the tip-link complex, a structure controlling actin polymerization in stereocilia. Proc Natl Acad Sci U S A. 2011;108(14):5825-5830.

17. Pepermans E, et al. The CD2 isoform of protocadherin-15 is an essential component of the tip- link complex in mature auditory hair cells. EMBO MolMed. 2014;6(7):984-992.

18. Richardson GP, de Monvel JB, Petit C. How the genetics of deafness illuminates auditory physiology. Annu Rev Physiol. 2011;73:311-334.

19. Vincent PF, Bouleau Y, Safieddine S, Petit C, Dulon D. Exocytotic machineries of vestibular type I and cochlear ribbon synapses display similar intrinsic otoferlin-dependent $\mathrm{Ca}^{2+}$ sensitivity but a different coupling to $\mathrm{Ca}^{2+}$ channels. J Neurosci. 2014;34(33):10853-10869.

20. Brandt A, Striessnig J, Moser T. CaV1.3 channels are essential for development and presynaptic activity of cochlear inner hair cells. J Neurosci. 2003;23(34):10832-10840.

21. Khimich D, et al. Hair cell synaptic ribbons are essential for synchronous auditory signalling. Nature. 2005;434(7035):889-894.

22. Johnson SL, Marcotti W, Kros CJ. Increase in efficiency and reduction in $\mathrm{Ca}^{2+}$ dependence of exocytosis during development of mouse inner hair cells. J Physiol (Lond). 2005;563(pt 1):177-191.

23. Vincent PF, Bouleau Y, Petit C, Dulon D. A synaptic F-actin network controls otoferlin-dependent exocytosis in auditory inner hair cells. Elife. 2015;4:e10988.

24. Brandt A, Khimich D, Moser T. Few CaV1.3 channels regulate the exocytosis of a synaptic vesicle at the hair cell ribbon synapse. J Neurosci. 2005;25(50):11577-11585.

25. Beurg M, et al. Control of exocytosis by synaptotagmins and otoferlin in auditory hair cells. J Neurosci. 2010;30(40):13281-13290.

26. Van Petegem F, Clark KA, Chatelain FC, 
Minor DL. Structure of a complex between a voltage-gated calcium channel beta-subunit and an alpha-subunit domain. Nature. 2004;429(6992):671-675.

27. Gregory FD, Bryan KE, Pangršič T, CalinJageman IE, Moser T, Lee A. Harmonin inhibits presynaptic Cav1.3 $\mathrm{Ca}^{2+}$ channels in mouse inner hair cells. Nat Neurosci. 2011;14(9):1109-1111.

28. Gregory FD, Pangrsic T, Calin-Jageman IE, Moser T, Lee A. Harmonin enhances voltage-dependent facilitation of Cav1.3 channels and synchronous exocytosis in mouse inner hair cells. J Physiol (Lond). 2013;591(13):3253-3269.

29. Liberman LD, Wang H, Liberman MC. Opposing gradients of ribbon size and AMPA receptor expression underlie sensitivity differences among cochlear-nerve/hair-cell synapses. J Neurosci. 2011;31(3):801-808.

30. Wong $\mathrm{AB}$, et al. Developmental refinement of hair cell synapses tightens the coupling of $\mathrm{Ca}^{2+}$ influx to exocytosis. EMBO J. 2014;33(3):247-264.

31. Pujol R, Puel JL. Excitotoxicity, synaptic repair, and functional recovery in the mammalian cochlea: a review of recent findings. Ann N $Y$ Acad Sci. 1999;884:249-254.

32. Geng R, et al. Modeling and preventing progressive hearing loss in Usher syndrome III. Sci Rep. 2017;7(1):13480.

33. Tian G, Lee R, Ropelewski P, Imanishi Y. Impairment of vision in a mouse model of Usher syndrome type III. Invest Ophthalmol Vis Sci. 2016;57(3):866-875.

34. Stölting $G$, et al. Direct interaction of $\mathrm{CaV} \beta$ with actin up-regulates L-type calcium cur- rents in HL-1 cardiomyocytes. J Biol Chem. 2015;290(8):4561-4572.

35. Mercer AJ, Chen M, Thoreson WB. Lateral mobility of presynaptic L-type calcium channels at photoreceptor ribbon synapses. J Neurosci. 2011;31(12):4397-4406.

36. Jing Z, et al. Disruption of the presynaptic cytomatrix protein bassoon degrades ribbon anchorage, multiquantal release, and sound encoding at the hair cell afferent synapse. J Neurosci. 2013;33(10):4456-4467.

37. Neef J, et al. The $\mathrm{Ca}^{2+}$ channel subunit $\beta 2$ regulates $\mathrm{Ca}^{2+}$ channel abundance and function in inner hair cells and is required for hearing. J Neurosci. 2009;29(34):10730-10740.

38. Mendus D, et al. Thrombospondins 1 and 2 are important for afferent synapse formation and function in the inner ear. Eur J Neurosci. 2014;39(8):1256-1267.

39. Yamada S, Nelson WJ. Synapses: sites of cell recognition, adhesion, and functional specification. Annu Rev Biochem. 2007;76:267-294.

40. Owald D, et al. Cooperation of Syd-1 with Neurexin synchronizes pre- with postsynaptic assembly. Nat Neurosci. 2012;15(9):1219-1226.

41. Fell B, et al. $\alpha 2 \delta 2$ Controls the function and transsynaptic coupling of Cav1.3 channels in mouse inner hair cells and is essential for normal hearing. J Neurosci. 2016;36(43):11024-11036.

42. Ness SL, et al. Genetic homogeneity and phenotypic variability among Ashkenazi Jews with Usher syndrome type III. J Med Genet. 2003;40(10):767-772.

43. Ebermann I, Wilke R, Lauhoff T, Lübben D,
Zrenner E, Bolz HJ. Two truncating USH3A mutations, including one novel, in a German family with Usher syndrome. Mol Vis. 2007;13:1539-1547.

44. Sadeghi M, Cohn ES, Kimberling WJ, Tranebjaerg L, Möller C. Audiological and vestibular features in affected subjects with USH3: a genotype/phenotype correlation. Int J Audiol. 2005;44(5):307-316.

45. Pan B, et al. Gene therapy restores auditory and vestibular function in a mouse model of Usher syndrome type 1c. Nat Biotechnol. 2017;35(3):264-272.

46. Emptoz A, et al. Local gene therapy durably restores vestibular function in a mouse model of Usher syndrome type 1G. Proc Natl Acad Sci U S A 2017;114(36):9695-9700.

47. Legendre K, Safieddine S, Küssel-Andermann P, Petit C, El-Amraoui A. $\alpha I I-\beta V$ Spectrin bridges the plasma membrane and cortical lattice in the lateral wall of the auditory outer hair cells. JCell Sci. 2008;121(pt 20):3347-3356.

48. Papal S, et al. The giant spectrin $\beta V$ couples the molecular motors to phototransduction and Usher syndrome type I proteins along their trafficking route. Hum Mol Genet. 2013;22(18):3773-3788.

49. Kamiya K, et al. An unusually powerful mode of low-frequency sound interference due to defective hair bundles of the auditory outer hair cells. Proc Natl Acad Sci U S A. 2014;111(25):9307-9312.

50. Le Calvez S, Guilhaume A, Romand R, Aran JM, Avan P. CD1 hearing-impaired mice. II. Group latencies and optimal f2/f1 ratios of distortion product otoacoustic emissions, and scanning electron microscopy. Hear Res. 1998;120(1-2):51-61. 